\title{
Unveiling the role of upper excited electronic states in the photochemistry and laser performance of anti- $\mathrm{B}_{18} \mathrm{H}_{22}$.
}

\author{
Luis Cerdán, ${ }^{1 *}$ Antonio Francés-Monerris, ${ }^{2,3}$ Daniel Roca-Sanjuán, ${ }^{*}{ }^{*}$ Jonathan Bould, ${ }^{5}$ Jiř́ Dolanský, ${ }^{5}$ \\ Marcel Fuciman, ${ }^{6}$ and Michael G. S. Londesborough ${ }^{5 *}$
}

${ }^{1}$ Institute of Physical Chemistry "Rocasolano", Consejo Superior de Investigaciones Científicas (CSIC), C/ Serrano 119, 28006, Madrid, Spain

${ }^{2}$ Université de Lorraine and CNRS, LPCT, F-54000 Nancy, France

${ }^{3}$ Departament de Química Física, Universitat de València, Dr. Moliner 50, 46100 Burjassot, Spain

${ }^{4}$ Institut de Ciència Molecular, Universitat de València, P.O.Box 22085, 46010 Valencia - Spain

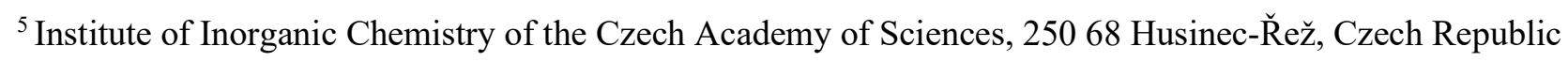

${ }^{6}$ Institute of Physics, Faculty of Science, University of South Bohemia, Branišovská 1760, 37005 České Budějovice, Czech Republic

*lcerdanphd@gmail.com; *Daniel.Roca@uv.es; *michaell@iic.cas.cz

\begin{abstract}
In the search for innovative new light sources, the discovery that solutions of the boron hydride anti- $\mathrm{B}_{18} \mathrm{H}_{22}$ generate photostable blue laser emission stands out in its significance as the first laser borane. Surprisingly, though, the laser performance of anti- $\mathrm{B}_{18} \mathrm{H}_{22}(\sim 10 \%$ efficiency) does not match the expectations based on its exceptional photophysical properties $\left(\Phi_{F}=0.97\right.$ and high photostability). To understand this contradiction, we herein present an investigation into the upper excited states of the anti- $\mathrm{B}_{18} \mathrm{H}_{22}$ photophysical system, which we suggest to be the most relevant factor to its laser performance. The use of computational quantum chemistry, laser and UV-vis spectroscopy, NMR spectroscopy, as well as Mass spectrometry, unveil the role of the upper excited states on the laser performance of anti- $\mathrm{B}_{18} \mathrm{H}_{22}$, showing that efficient excited state absorption (ESA) leads to the population of these states, and results not only in the loss of laser efficiency, but also in the activation of chemically reactive relaxation pathways and the formation of photochemically produced novel molecular species. The likely composition of these photoproducts, formed upon prolonged high intensity laser irradiation, are inferred from their molecular masses, NMR properties, and calculated natural orbitals. Together, these results are of key importance to the complete understanding of the anti- $\mathrm{B}_{18} \mathrm{H}_{22}$ photophysical system and provide valuable information to chemists and laser physicists working to mitigate deficiencies and enhance the performance of the next generation of borane lasers and borane-based photoactive materials.
\end{abstract}




\section{Introduction}

Boron hydrides (commonly referred to as boranes) form deltahedral cluster molecules. As chemical and physical behaviour are often a function of structure, then the boranes have, with their unique molecular geometries, a large potential for novel properties. ${ }^{1}$ We are interested in applying this general principle to the interaction of borane clusters with light, and a number of recent discoveries and developments (welldocumented in review articles ${ }^{2}$ ) have shown the vast potential of these inorganic species as drivers of innovation in contemporary optical materials. Within this area, our focus has been on the macropolyhedral borane cluster, anti- $\mathrm{B}_{18} \mathrm{H}_{22}$, which we have shown to be a highly photostable emitter of blue light with fluorescence quantum yields approaching unity. ${ }^{3}$ Furthermore, via various substitutions to its 18 -vertex boron skeleton, derivatives of anti- $\mathrm{B}_{18} \mathrm{H}_{22}$ provide, for example, environment-sensitive thermochromic luminescence, ${ }^{4}$ single-molecule multiple emissions, ${ }^{5}$ and the photosensitization of oxygen. ${ }^{6}$ However, our central aim in this growing field continues to be innovation in laser emission from these unusually structured inorganic molecular species.

Amongst the fluorescent boranes reported so far, anti- $\mathrm{B}_{18} \mathrm{H}_{22}$ stands out not only for its excellent emission properties but for sustaining laser emission. ${ }^{7}$ Solutions of anti- $\mathrm{B}_{18} \mathrm{H}_{22}$ thus emit, under pulsed excitation, violet-blue (407 nm) laser light with an efficiency (ratio of output/input energies) of 9.5\%, and a photostability superior to many of the commercially available state-of-the-art blue laser dyes. ${ }^{7}$ Although this demonstration opened the doors to the development of a whole new class of laser materials based on a previously untapped resource for laser technology - the boranes - there are still issues in its performance that prevent the exploitation of their full potential. These deficiencies lead to surprising outcomes when compared to competitive laser materials. For example, that 4,4'-diphenylstilbene (DPS), an organic laser dye with similar absorption and emission frequencies as anti- $\mathrm{B}_{18} \mathrm{H}_{22}$ but with an inferior fluorescence quantum yield of 0.74 , gave laser efficiencies better than that of anti- $\mathrm{B}_{18} \mathrm{H}_{22} .{ }^{7}$ Also, since the discovery of lasing from anti- $\mathrm{B}_{18} \mathrm{H}_{22}$, no further reports of other fluorescent boranes displaying laser emission have appeared despite other fluorescent borane species with comparable photophysical characteristics having been made. ${ }^{4-6,8,9}$ In this sense, the ability of anti- $\mathrm{B}_{18} \mathrm{H}_{22}$ to function as a laser seems to be the exception rather than the rule with regard to luminescent boranes. All these considerations raise the question of what might be the main factors mitigating the development of borane-based lasers. From previous work on anti- $\mathrm{B}_{18} \mathrm{H}_{22},{ }^{7}$ the observation of a reduction in luminescence quantum yield due to concentration quenching, and the increase in scattering losses due to solubility problems, cannot by itself fully explain the weakness in its laser emission or the absence of other boranes sustaining laser emission. Therefore, other sources of losses must be at play, and it is the intention of this contribution to delineate them.

Within the contemporary understanding of laser science, one photophysical phenomenon that can lead to the reduction in laser efficiency, and even preclude it altogether, is the activation of losses channels through the promotion of the laser molecule to upper non-lasing excited states via excited state absorption (ESA) of the emission or pump light. ${ }^{10}$ Our hypothesis is that this is the case for anti- $\mathrm{B}_{18} \mathrm{H}_{22}$, and therefore of primary 
importance to the future development of borane laser technology is a thorough understanding of the role that the upper excited states of anti- $\mathrm{B}_{18} \mathrm{H}_{22}$ plays in its laser performance. The findings of our combined experimental-computational approach, reported in this manuscript, represent a first but fundamental step to this ultimate goal.

\section{Results}

\subsection{Ground and excited state photophysics}

In the original paper describing the photophysics of anti- $\mathrm{B}_{18} \mathrm{H}_{22},{ }^{3}$ the fluorescence quantum yield $\left(\Phi_{F}=\right.$ 0.97 ) and life-time ( $\tau=11.2 \mathrm{~ns}$ ), together with the steady-state Ground State Absorption (GSA) and Stimulated Emission (SE) spectra, as well as the femtosecond Transient Absorption (TA) spectrum below $2.75 \mathrm{eV}$, were measured. To this original data is added a new TA measurement, using equipment that covers a wider spectrum. This TA spectrum was acquired for a delay of $15 \mathrm{ps,} \mathrm{enough} \mathrm{time} \mathrm{for} \mathrm{the} \mathrm{molecule} \mathrm{to} \mathrm{thermalize}$ and reach the excited state minimum. All these spectra are included in Figures 1a and $1 \mathrm{~b}$ expressed as cross sections; a magnitude that is preferred in the context of laser physics (see Methods for conversion details).

To support these experimental observations a theoretical analysis was performed based on the completeactive-space self-consistent field / complete-active-space second-order perturbation theory (CASSCF/CASPT2) methodology (see Methods), which is a multiconfigurational (electron-correlation) quantum chemistry approach for computational investigation of electronic states in molecular systems. ${ }^{11}$ The calculated vertical absorption energies and associated oscillator strengths of all the $\mathrm{S}_{0} \rightarrow \mathrm{S}_{1}(\mathrm{GSA}), \mathrm{S}_{1} \rightarrow \mathrm{S}_{0}$ $(\mathrm{SE})$, and $\mathrm{S}_{1} \rightarrow \mathrm{S}_{\mathrm{N}}$ (Excited State Absorption, ESA) allowed transitions are displayed in Figure 1c and collected into Table S1. To make the comparison with the experimental results easier, the oscillator strength values and vertical energies were convolved into spectral bands (see Methods). The computed GSA, SE and ESA crosssection spectra are displayed as well in Figure 1c. Below $3.5 \mathrm{eV}$, where the GSA is negligible, the TA spectrum is given by the difference between the ESA and SE spectra $\left(\sigma_{T A}=\sigma_{E S A}-\sigma_{S E}\right) .{ }^{12,13}$ The measured and computed TA cross section spectra are displayed in Figure $1 \mathrm{~b}$. 
a)

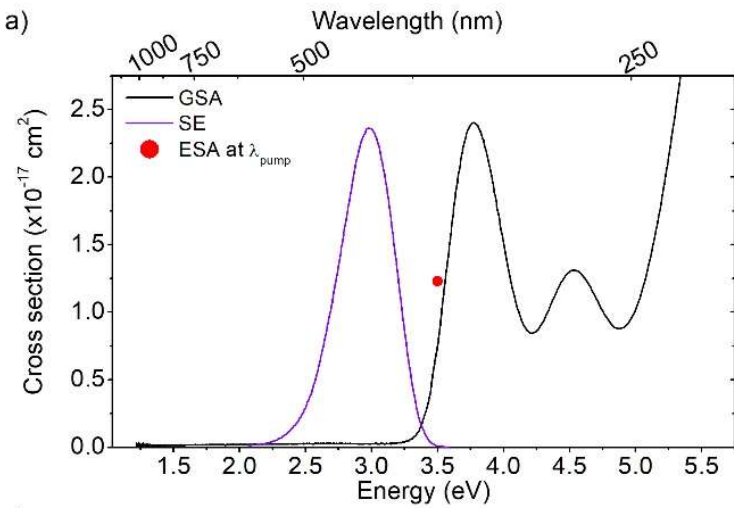

c)

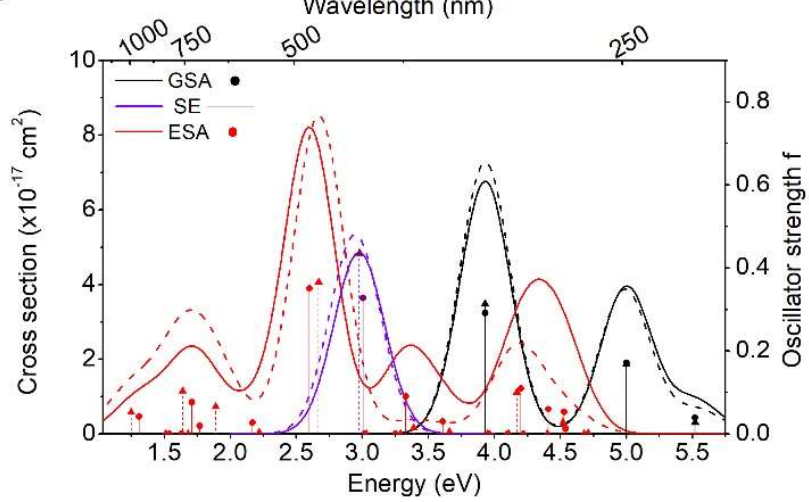

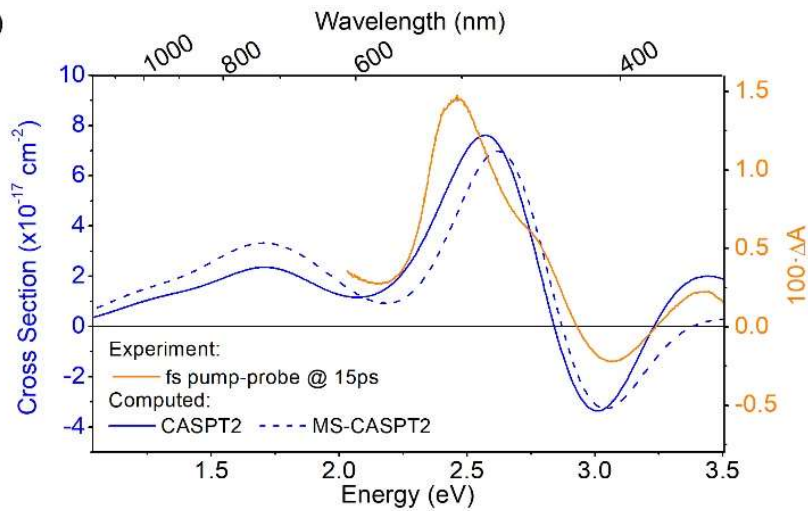

d)

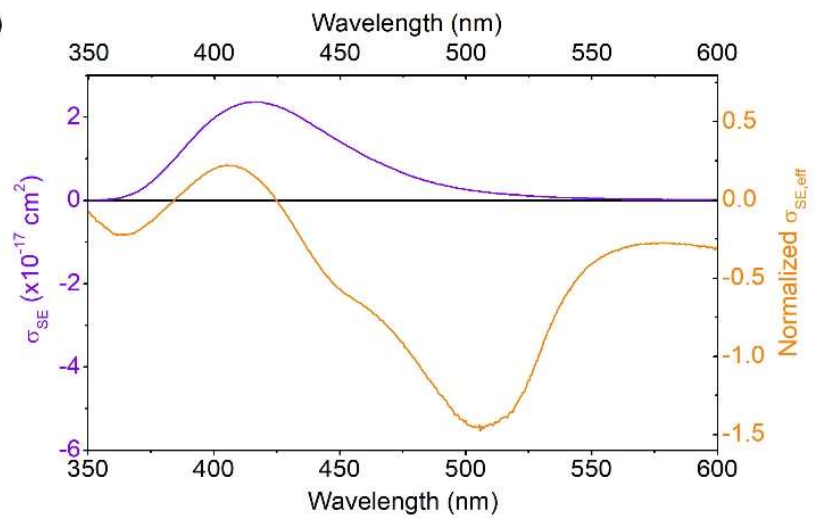

Figure 1: a) Measured Ground State Absorption (GSA) and Stimulated Emission (SE) spectra, and Excited State Absorption (ESA) at $\lambda_{\text {pump }}=355 \mathrm{~nm}(3.5 \mathrm{eV})$ for anti- $\mathrm{B}_{18} \mathrm{H}_{22}$ in $c$-hexane solution. b) Measured (15 ps delay) and computed Transient Absorption (TA) spectra for anti- $\mathrm{B}_{18} \mathrm{H}_{22}$. c) Computed oscillator strengths (scatters and vertical lines) and cross-sections (curves) for GSA, SE and ESA . d) Experimental SE ( $\left.\sigma_{S E}\right)$ (purple curve) and effective SE ( $\sigma_{S E, e f f}$ ) (orange curve) spectra. In b) and c) solid and dashed lines display, respectively, CASPT2 and MS-CASTP2 calculation.

As seen in Figure 1, there is a general blue-shift in the computed allowed transitions with respect to the experimental measurements. The weaker GSA bands, however, suffer a higher discrepancy. These deviations could be a consequence of solvent or vibronic effects, ${ }^{14}$ which were not included in the computations. In addition, the calculation overestimates (in absolute value) the TA at the emission band (Fig. 1a, $3 \mathrm{eV}$ ). The reason for this discrepancy is that the calculations use the geometry of a molecule of anti- $\mathrm{B}_{18} \mathrm{H}_{22}$ that has completely relaxed into the "cold" excited-state minimum. That is to say that the molecule has had sufficient time to reach a perfect Bolzmann distribution of the excited-state minimum. In the experimental setting, however, the molecule reaches the excited state minimum but still keeps a certain degree of kinetic energy and momentum. In other words, the calculation renders the TA spectrum of the "cold" excited state, while the experiment reveals the spectrum of the "hot" excited state. In any case, there is generally a good agreement in the band positions and, most importantly, in the cross-section values, which are of the same order of magnitude. This agreement is fundamental for the following discussions, as the computed spectra not only will fill in the gap left by the experiments, but will give access to information not experimentally attainable.

Finally, to supplement the experimental picture, the ESA at the laser pump wavelength $\left(\lambda_{\mathrm{p}}=355 \mathrm{~nm}\right.$, $3.5 \mathrm{eV}$ ) shown in Figure 1a was also measured making use of the saturable absorption behaviour of the $\mathrm{S}_{0}-\mathrm{S}_{1}$ 
transition. This was retrieved by means of intensity dependent transmission measurements ${ }^{12,15}$ together with adequate numerical simulations (see Supplementary Material). Using this procedure, in the absence of ESA, the light transmission should increase with the pump intensity as a consequence of the ground state population depletion induced by the strong pumping. The presence of ESA would counterbalance this effect, and might even reduce it (i.e. decrease in transmission) if the ESA were strong enough. The experimental results show that for anti- $\mathrm{B}_{18} \mathrm{H}_{22}$ there is a decrease in transmission with the pump intensity (Figure 2), indicating that the ESA at the pump wavelength is rather substantial. Indeed, the numerical analysis shown in Figure 2 suggests that the ESA cross section $\left(\sigma_{l p}\right)$ is a $65 \%$ stronger than the GSA one $\left(\sigma_{\sigma_{p}}\right)$. Interestingly, this excess of ESA reveals that anti- $\mathrm{B}_{18} \mathrm{H}_{22}$ is a "reverse saturable absorber" that could be exploited in optical limiting applications. ${ }^{16}$ Unfortunately, however, this same excess has dramatic consequences for the laser performance, as will transpire later on. The value of the ESA cross section at the pump wavelength has been included in Figure 1a (red dot) for comparison purposes.

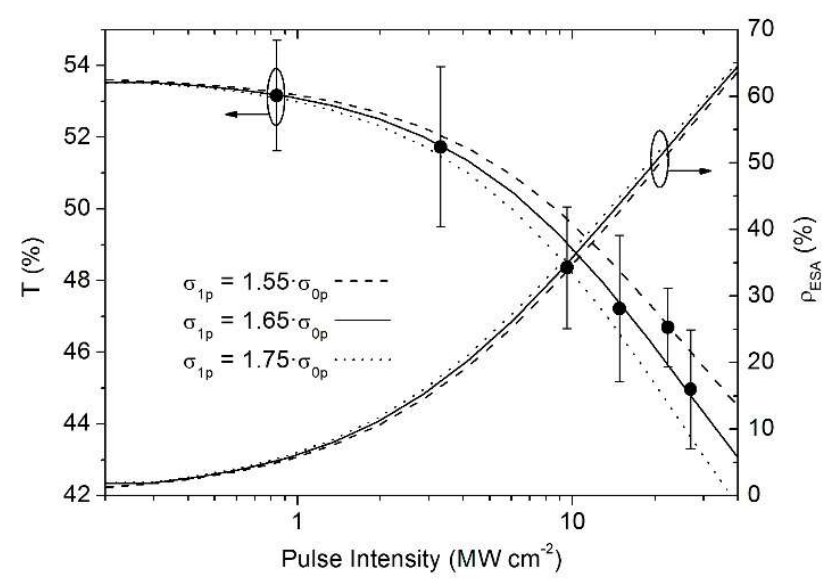

Figure 2: Intensity dependent pump transmission $\mathrm{T}$ (left) and percentage of absorbed photons that goes into ESA $\rho_{E S A}$ (right) for a $0.4 \mathrm{~cm}$ path-length $0.5 \mathrm{mM}$ anti- $\mathrm{B}_{18} \mathrm{H}_{22} / \mathrm{c}$-hex dissolution. Circles are experimental data. Error bars indicate the standard deviation of the mean values. The curves are calculated as described in the text and supplementary material.

\subsection{Influence of ESA on the laser performance of anti-B ${ }_{18} \mathrm{H}_{22}$}

The laser performance of any laser device strongly depends on the cavity design and the properties of the active material. ${ }^{10 b}$ Focusing on the latter, the amplification capability of any active material, referred to as its optical gain $g,{ }^{12,17,18}$ is defined approximately as:

$$
g=\sigma_{S E, \text { eff }} \Delta n=\left(\sigma_{S E}-\sigma_{E S A}\right) \Delta n
$$

where $\Delta n$ is the population inversion between the laser levels and $\sigma_{S E, \text { eff }}$ is the effective stimulated emission cross section, which is the difference between the SE and ESA cross sections. 
Another process fundamental to laser performance is the pump to laser energy conversion $\eta_{p}$, which accounts for the probability that an absorbed pump photon contributes to the available population inversion $\Delta n .^{10 \mathrm{c}}$

It is established that ESA degrades the laser performance through reductions in $\sigma_{S E, \text { eff, }}, \Delta n$, and/or $\eta_{p}$. An inspection of equation (1) reveals that the ESA at the emission region has an immediate negative impact on the optical gain and on the laser performance, as it reduces $\sigma_{S E, \text { eff. }}{ }^{12,17-21}$ In relation to anti- $\mathrm{B}_{18} \mathrm{H}_{22}$, the experimental TA spectrum (Figure 1b) shows a strong ESA band centred around $450 \mathrm{~nm}$, which matches excellently to the calculated ESA resonance at $464 \mathrm{~nm} / 2.67 \mathrm{eV}$, corresponding to the transition $\mathrm{S}_{1} \rightarrow \mathrm{S}_{8}$ (Table $\mathrm{S} 1$ ), with the high oscillator strength of 0.3658 , comparable in magnitude to that for the $\mathrm{S}_{0} \rightarrow \mathrm{S}_{1}$ (GSA). ${ }^{3}$ Furthermore, this ESA clearly overlaps with the SE spectrum (Figure 1c). In fact, the overlap is so large, and the ESA cross section is so strong, that $\sigma_{S E, \text { eff }}$ is substantially diminished and distorted as compared with $\sigma_{S E}$ (Figure 1d). Not only the maximum cross section (and gain) is strongly reduced, but there are regions in which $\sigma_{S E, \text { eff }}$ becomes negative, meaning that the medium absorbs, instead of amplifying, the generated laser light. Thus, for anti- $\mathrm{B}_{18} \mathrm{H}_{22}$ the presence of ESA at the emission region entails both a weaker amplification capability (i.e., lower efficiency/higher threshold) and a smaller laser wavelength tuning range, ${ }^{22}$ as the region where $\sigma_{S E, \text { eff }}>0$ is effectively narrowed (Figure 1d).

Another mechanism that it is known to have a negative impact on the laser performance is the so-called "singlet-singlet annihilation" (SSA), a non-radiative process in which an excited molecule $A$ transfers its energy through a dipole-dipole long range interaction to another excited molecule $B \cdot{ }^{23-25}$ Consequently, $A$ relaxes non-radiatively to the ground state and $B$ is promoted to an upper excited state, $\mathrm{S}_{\mathrm{N}}$. As a result, the system ends up with only one excited molecule when it started with two. In other words, there is a reduction in the population of the first excited state (upper laser level) and thus in the population inversion $\Delta n$ needed for lasing. This entails a reduction in the gain (equation (1)) and, ultimately, in the laser efficiency. The SSA in solutions is analogous to the Förster Resonance Energy Transfer (FRET) between an excited donor and an acceptor in the ground state, ${ }^{24,26}$ only that in SSA both donor and acceptor are in the excited state (two excited molecules of anti- $\mathrm{B}_{18} \mathrm{H}_{22}$ in this case). For both FRET and SSA to take place two main conditions must be fulfilled: a) the absorption spectrum of the acceptor (ESA for SSA) must overlap with the fluorescence spectrum of the excited donor; and b) the donor and acceptor (excited molecules for SSA) must be close enough to exchange the energy. A parameter that helps in quantifying the extent of overlap of the emission and absorbance spectra (condition a), and how close is close enough (condition b), is the Förster Radius $R_{0}$, which is the molecular separation for which the probability of donor emission and the probability of energy transfer (to the acceptor) is equal. The transfer rate for a SSA interacting pair is given by:

$$
k_{S S A}=\tau^{-1}\left(R_{0} / r\right)^{6}
$$

where $\tau$ is the fluorescence life-time and $r$ is the molecular separation. Thus, for excited molecules that are separated by distances above twice or thrice $R_{0}$, SSA becomes negligible. In contrast, for separations below 
$R_{0}$, SSA becomes dominant.

Figure 1c shows that for anti- $\mathrm{B}_{18} \mathrm{H}_{22}$ condition a) is fulfilled, as there is a substantial overlap between the ESA spectrum (acceptor) and the SE spectrum (donor). The computed Förster Radius for SSA in anti$\mathrm{B}_{18} \mathrm{H}_{22}$ is close to $R_{0}=4 \mathrm{~nm}$ (details in Methods section), a value that it is on par with the values found for other FRET pairs. ${ }^{4 a, 27}$ This means that SSA will be highly effective (and detrimental for laser emission) if the separation between excited molecules of anti- $\mathrm{B}_{18} \mathrm{H}_{22}$ is short enough (less than $8 \mathrm{~nm}$ ). The high concentrations and the strong pumping conditions in the laser experiments guarantee that this condition is usually fulfilled. However, it is noteworthy that in our femtosecond TA experiments, using pump intensities of around $10^{14}$ photons per pulse, per $\mathrm{cm}^{2}$, we do not observe any hint of the annihilation processes, suggesting a significant energy threshold before SSA becomes relevant.

Other transfer processes affecting the laser efficiency are the singlet-triplet and triplet-triplet annihilation mechanisms, ${ }^{26}$ but they can be ruled out for anti- $\mathrm{B}_{18} \mathrm{H}_{22}$, as its quantum yield of triplet state generation is below $0.03 \%{ }^{3}$

Finally, the ESA at the pump wavelength affects the pump to laser energy conversion $\eta_{p}$ which, in turn, significantly reduces the laser efficiency. ${ }^{12,21,28-31}$ The pump photons "trap" the excited molecules in a cycle between the $S_{1}$ and $S_{N}$ states, in such a way that the molecules can absorb several pump photons before relaxing radiatively to the ground state. This process, known as 'photoquenching', leads to an effective reduction in luminescence quantum yield under high intensity laser irradiation. ${ }^{31-32}$ ESA measurements (Fig. 1b) for anti$\mathrm{B}_{18} \mathrm{H}_{22}$ show absorption between $340 \mathrm{~nm}-380 \mathrm{~nm}$, which is supported by our theoretical results that compute an ESA resonance at $3.4 \mathrm{eV} / 365 \mathrm{~nm}$ (Figure $1 \mathrm{~b}$ and 1c). Calculations also suggest that this ESA, covering the region of the pump wavelength, results in $\mathrm{S}_{1} \rightarrow \mathrm{S}_{11}$ and $\mathrm{S}_{1} \rightarrow \mathrm{S}_{12}$ transitions (Table $\mathrm{S} 1$ ), that compete with the $\mathrm{S}_{1} \rightarrow \mathrm{S}_{0}$ stimulated emission pathway. Indeed, the experimentally measured ESA cross section at $\lambda_{\mathrm{p}}(355 \mathrm{~nm}$, $3.5 \mathrm{eV}$ ), inferred by means of intensity dependent transmission measurements (Figure 1a and 2), is around a $65 \%$ stronger than the GSA at the same wavelength. Such levels of ESA cross sections have been shown to result in extreme reductions in laser efficiency of transversely pumped lasers. ${ }^{28-30}$ Indeed, the level of ESA for anti- $\mathrm{B}_{18} \mathrm{H}_{22}$ at the pump wavelength is such that there could be statistically more pump photons absorbed in the excited states than in the ground state. In fact, the percentage of absorbed photons that goes into ESA $\rho_{E S A}$ (see Equation (S8) of Supplementary Information) may surpass $60 \%$ at the highest pump intensities in the intensity dependent transmission experiment (Figure 2). To assess the laser performance of anti- $\mathrm{B}_{18} \mathrm{H}_{22}$,

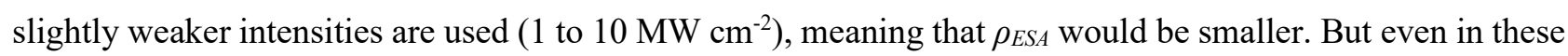
milder pumping conditions, a significant number of absorbed pump photons are wasted and do not contribute to population inversion neither, ultimately, to the laser process.

\subsection{Influence of ESA on the photodegradation of anti-B ${ }_{18} \mathrm{H}_{22}$}

The electron-deficiency of boron (it has one valence electron fewer than its number of valence orbitals) requires the boranes to share electron density in multi-centred delocalized cluster assemblies. ${ }^{33}$ The 
characteristic 3-centre-2-electron deltahedral connectivities of the boranes have stronger $\sigma$ character than aromatic organic compounds ${ }^{34}$ and, therefore, might be expected to form highly photostable structures in comparison to hydrocarbons. This is, indeed, the case for anti- $\mathrm{B}_{18} \mathrm{H}_{22}$, which displays a photostability under laser irradiation superior to many of the commercially available state-of-the-art blue laser dyes. ${ }^{7}$ Nevertheless, this superior photostability over organic compounds is not as overwhelming as we had anticipated based on the fundamentals of its bonding characteristics, which suggested to us that perhaps the ESA photophysics of anti- $\mathrm{B}_{18} \mathrm{H}_{22}$ effects its own photodegradation.

Increasing the population of upper excited states in any molecule can lead to the activation of chemically reactive deexcitation pathways. Molecular laser materials are no exception, and can result in the photodegradation of the active molecules and to the reduction of the laser efficiency after extended irradiation times. ${ }^{10 a, 35}$ In our previous work describing the laser properties of $c$-hexane solutions of anti- $\mathrm{B}_{18} \mathrm{H}_{22}$ prepared in a glove box and sealed under argon, we had detected the generation of bubbles if high pump intensities were used. ${ }^{7}$ This bubbling was accompanied, after continuous $355 \mathrm{~nm}$ laser excitation by the deposition of white markings at the quartz sample cuvette/solvent interface (in the area where the laser excitation beam contacts the solution - see Figure 3) presumably due to a light-induced chemical interaction between the sample and the cuvette surface and/or a laser ablation process. 

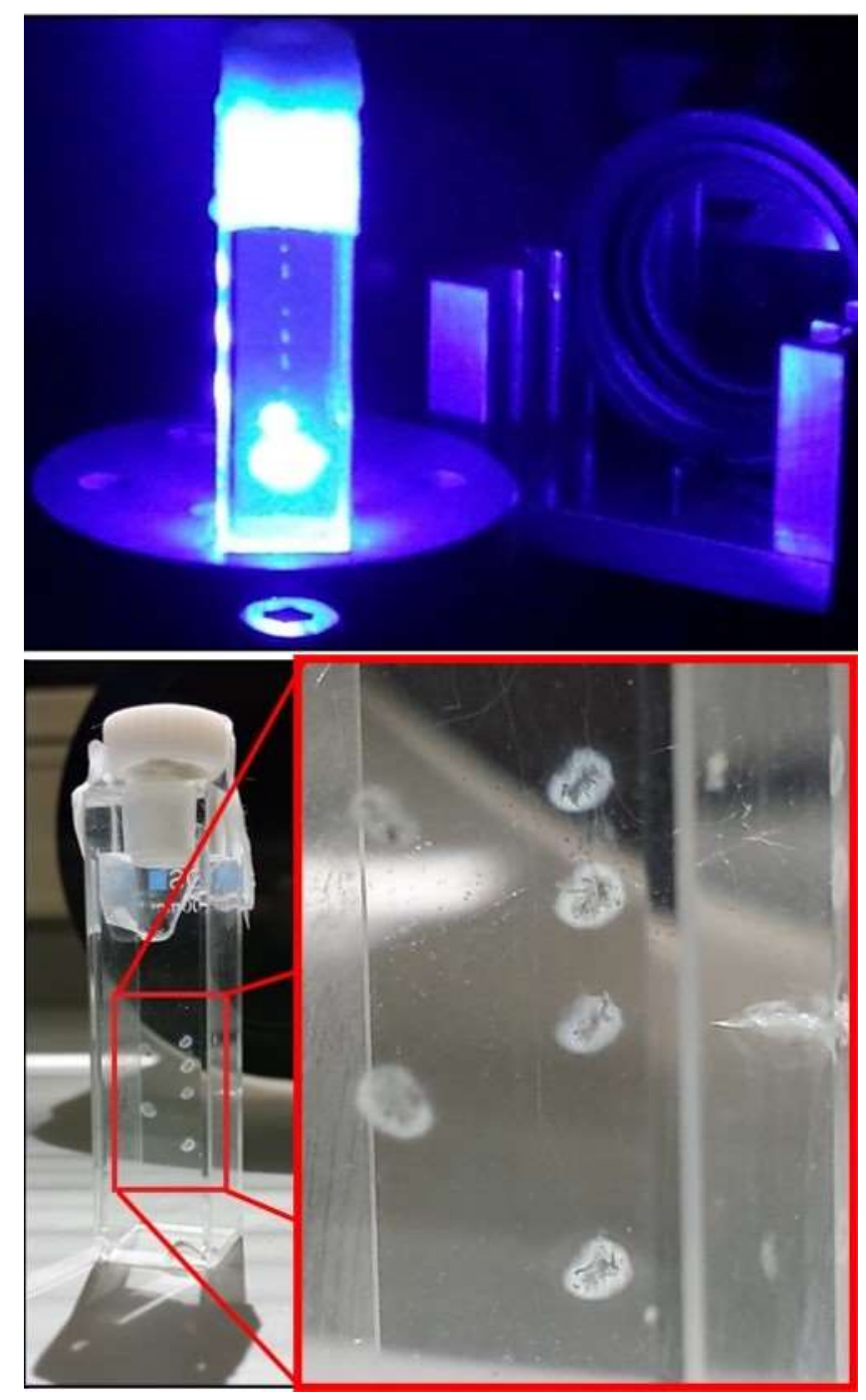

Figure 3. Frontal view of Laser Induced Fluorescence experiment (top) showing a stream of bubbles emanating from the laser spot, and (bottom) photographs of sample cuvette showing white deposits after removal of all solution after 210 minutes of continuous laser excitation.

These white deposits eventually partially blocked the pump radiation, thus reducing the stimulated emission from the sample, and initially leading us to misread the photostability of the gain material. An NMR analysis (see Fig. S1) showed that these white deposits, which readily dissolved in deuterated acetonitrile, were composed solely of boric acid, $\mathrm{B}(\mathrm{OH})_{3}$ - a usual product of complete oxidation of borane compounds, easily identifiable in the ${ }^{11} \mathrm{~B}$ NMR spectrum as a single peak at $19 \mathrm{ppm}$. In the absence of air, the formation of $\mathrm{B}(\mathrm{OH})_{3}$ is conceivably brought about by the reaction of anti- $\mathrm{B}_{18} \mathrm{H}_{22}$ with the quartz cuvette surface, driven by the intense UV irradiation from the pump laser. Consequently, we changed the experimental set-up, using a prism to direct the pump beam at right-angles into the sample cuvette from above, thus avoiding irradiation of the sample at the solution/cuvette wall interface (see Fig. S2 and Methods). These precautions allowed us to focus exclusively on the laser/anti- $\mathrm{B}_{18} \mathrm{H}_{22}$ interaction.

In our study, we irradiated $1 \mathrm{mM} c$-hexane solutions of anti- $\mathrm{B}_{18} \mathrm{H}_{22}$ at $355 \mathrm{~nm}$ with $12 \mathrm{~mJ}$ pulses, taking samples for analysis at regular intervals up to a total experimental time of 210 minutes. This pump energy 
affords a pump intensity of $24 \mathrm{MW} \mathrm{cm}^{-2}$, enough to promote a vast number of molecules to the $\mathrm{S}_{\mathrm{N}}$ state (Fig. 2). In total, six samples were collected and labelled in reference to their individual irradiation times and energies received, as: $0 \operatorname{mins}(0 \mathrm{MJ}), 15 \mathrm{mins}(0.11 \mathrm{MJ}), 30 \mathrm{mins}(0.22 \mathrm{MJ}), 60 \operatorname{mins}(0.43 \mathrm{MJ}), 120 \operatorname{mins}(0.86 \mathrm{MJ})$, and 210mins(1.51 MJ). Figure S3 reveals a visible and gradual opaqueness and yellowing developing from the 0 mins to the 210 mins sample. This trend is perhaps more evident from Figure S4 in which samples 0mins and 210mins are directly compared, and it shows, in the case of the $210 \mathrm{mins}(1.51 \mathrm{MJ})$ sample, a very fine white particulate matter in suspension and a distinctive yellowish hue. All samples showed blue-purple luminescence under a UV lamp, although not of equal intensity. A visual comparison of the samples 0mins, 150mins and 210mins in quartz NMR tubes placed next to each other may be seen in Figure S5. This photograph shows an empirical reduction in fluorescence intensity in the $210 \mathrm{mins}$ sample, and points to the formation of absorptive but barely- to non-emissive photoproducts of anti- $\mathrm{B}_{18} \mathrm{H}_{22}$ upon high intensity irradiation.

To elucidate and quantify these changes, a small aliquot of each irradiated solution was extracted to conduct UV-vis photophysical measurements. The remaining solutions were subsequently subjected to NMR spectroscopic and mass spectrometric measurements.

Figure 4a shows the evolution of the absorption spectra with respect to the irradiation time. Even after only 15 minutes of intense irradiation, there are measurable changes in the absorption signatures, with a slight reduction in the strength of all absorption bands and a slight increase in the absorption strength of the interband "valleys". The longer the samples are irradiated, the more evident these modifications become. These changes, which are ascribed to the formation of photoproducts with absorption properties unlike those of anti$\mathrm{B}_{18} \mathrm{H}_{22}$, are more clearly shown in the differential absorbance plotted in Figure $4 \mathrm{~b}$. In addition, a new absorption contribution can be seen arising in this plot at around $370 \mathrm{~nm}$ that tapers to $450 \mathrm{~nm}$. 
a)
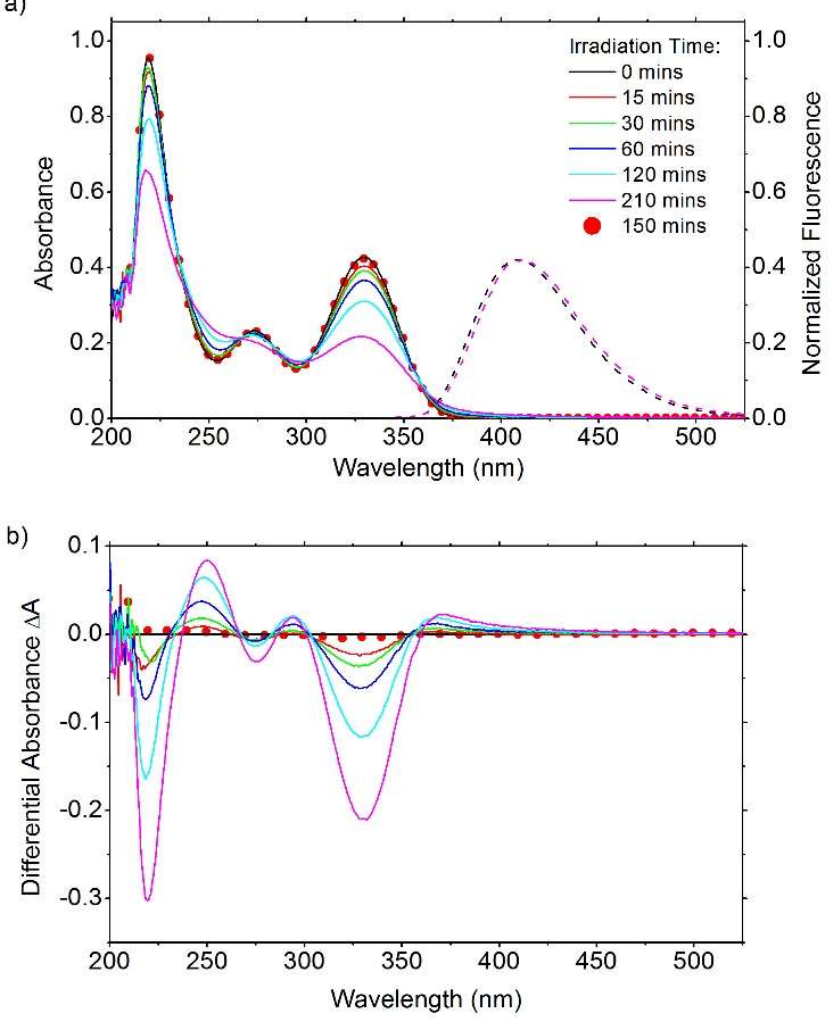

c)

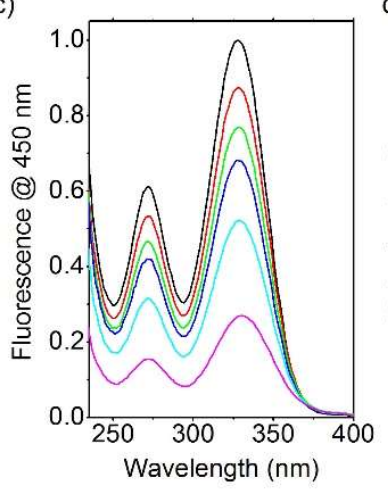

d)
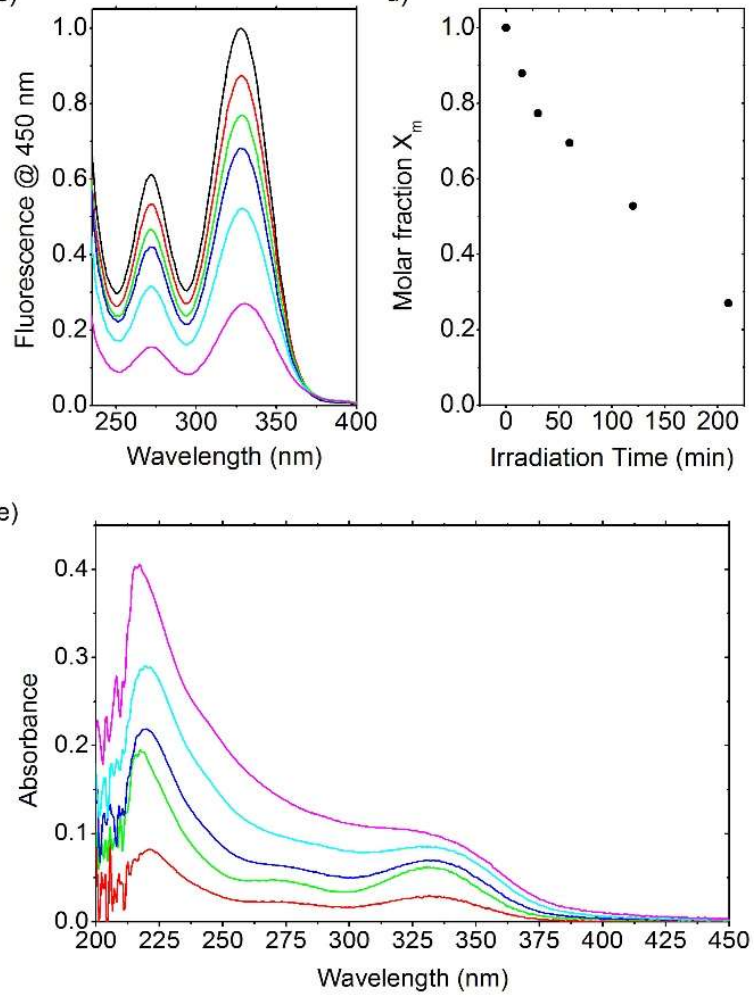

Figure 4: Evolution of UV-vis photophysics with irradiation time: a) Absorption spectra (solid lines) and normalized fluorescence spectra (dashed lines; $\left.\lambda_{\mathrm{ex}}=330 \mathrm{~nm}\right)$. b) Differential absorbance $\left(\Delta \mathrm{A}=\mathrm{A}\left(t_{\text {irr }}\right)-\mathrm{A}(0)\right)$. c) Excitation spectra $\left(\lambda_{\mathrm{em}}=450 \mathrm{~nm}\right)$. d) Calculated molar fraction of surviving anti- $\mathrm{B}_{18} \mathrm{H}_{22}$. e) Calculated photoproduct absorption spectra. The colour legend in a) applies to b), c), and e). The absorbance values in a) and e) correspond to $1 \mathrm{~mm}$ path-length cuvettes.

The fluorescence spectra of the reference $(0$ mins $)$ and the longest irradiated sample (210 mins) are almost identical (Figure 4a). There is a $3 \mathrm{~nm}$ red-shift in the irradiated sample fluorescence spectrum that could be ascribed to environmental effects induced by the chemical reactions taking place upon the formation of photoproducts (change in acidity, polarity, refractive index, etc). Nevertheless, the similarity in the shape of the spectra suggests that any photoproducts must be non-emissive (or, considerably less likely, coincidentally emissive at the same wavelength) and that all the observed fluorescence is therefore most probably from the remaining anti- $\mathrm{B}_{18} \mathrm{H}_{22}$. This conclusion is confirmed by the manner in which the excitation spectra evolve with respect to the irradiation time (Figure 4c), which show a reduction in the overall signal but no modification in the shapes of the spectra.

Figure 5 shows a stacked plot of the ${ }^{11} \mathrm{~B}-\left\{{ }^{1} \mathrm{H}\right\}$ NMR spectra for the samples irradiated from 15 to 210 minutes (see Figures S6-S11 for individual spectra). The spectrum for the 0mins reference sample, shown in Figure S6, presents the expected nine peaks of equal intensity that are characteristic of the centrosymmetrical 18-vertex borane cluster of anti- $\mathrm{B}_{18} \mathrm{H}_{22}$, the NMR properties of which are well known. ${ }^{36}$ Samples $15 \mathrm{mins}$ and 30 mins show almost no change to that of the reference. Indeed, it is only after 60 minutes of irradiation time 
that new signals can be seen clearly on the base-line of the spectrum (Fig. 5), suggesting that for the first hour of irradiation (a total energy of $0.43 \mathrm{MJ}$ ), the degradation of anti- $\mathrm{B}_{18} \mathrm{H}_{22}$ to other boron-containing species is negligible. By 210 mins, however, there is a clutter of new peaks, easily distinguishable from the nine resonances belonging to the remaining anti- $\mathrm{B}_{18} \mathrm{H}_{22}$. A crude integration of the respective peaks (i.e. an integration of the nine resonances for anti- $\mathrm{B}_{18} \mathrm{H}_{22}$ compared to an integration of all the remaining peaks) suggests that by 210 mins roughly $50 \%$ of the anti- $\mathrm{B}_{18} \mathrm{H}_{22}$ has degraded into other boron hydride species. It is notable that the new peaks in the NMR are consistent for novel cluster borane species and not simply boric acid. We discuss later on what might be the constitution of these photoproducts, but for the meantime it is sufficient to say that these NMR data are broadly consistent with the trend observable in UV-vis spectra shown in Figure 4.

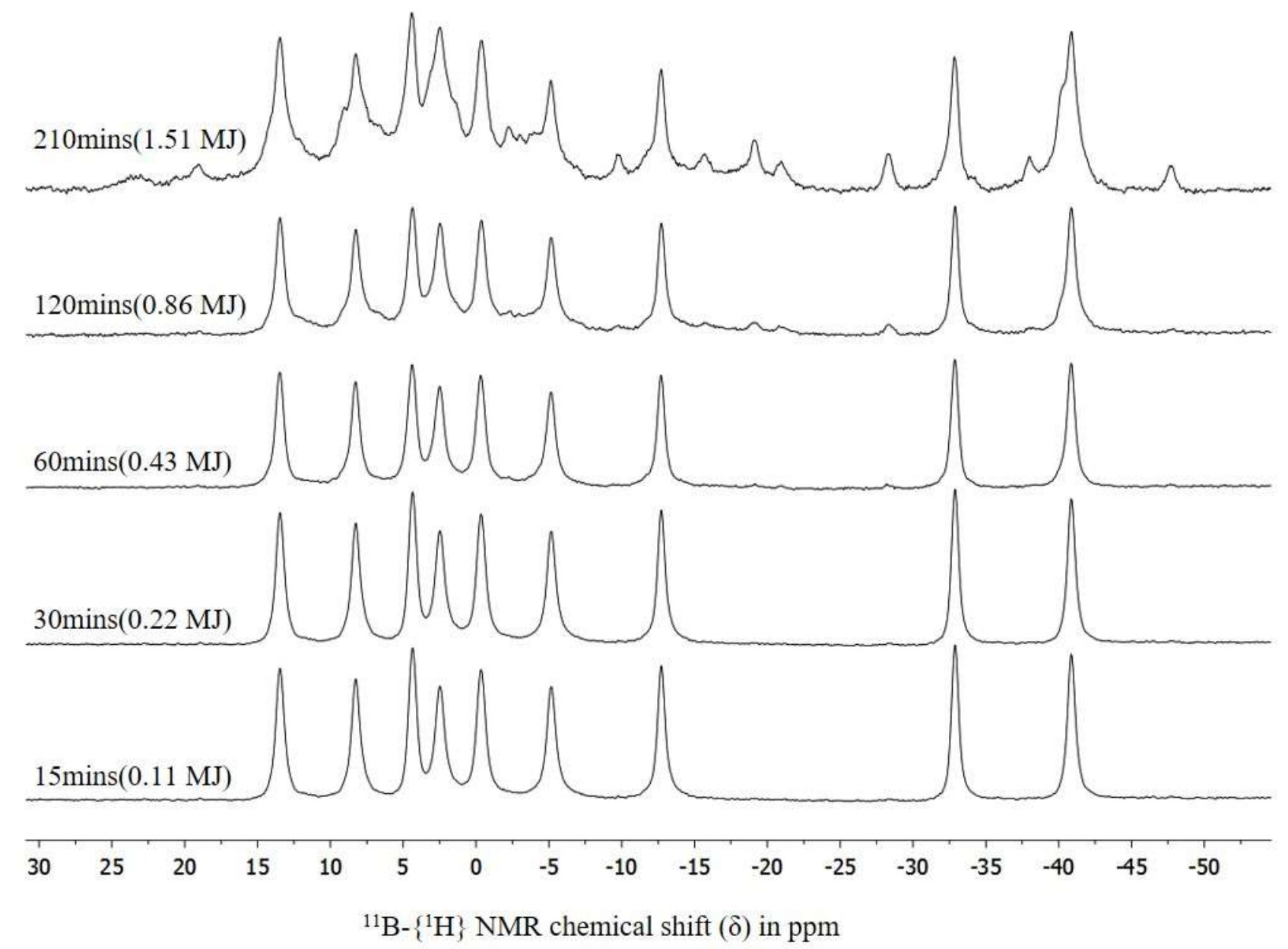

Figure 5. stacked plot of the ${ }^{11} \mathrm{~B}-\left\{{ }^{1} \mathrm{H}\right\}$ NMR spectra for the samples irradiated from 15 to 210 minutes

Figure $4 \mathrm{~d}$ shows the calculated molar fraction (see Methods) of surviving anti- $\mathrm{B}_{18} \mathrm{H}_{22}$ molecules as a function of irradiation time, which follows a near exponential decay path, culminating after 210 minutes with less than a $30 \%$ of anti- $\mathrm{B}_{18} \mathrm{H}_{22}$ molecules remaining intact. This figure compares reasonably to that inferred from the measured NMR data of approximately $50 \%$ degradation. Either way, both of these indirect methods suggest significant photodegradation, and with a good estimation of the molar fraction, the absorbance 
spectrum of the photoproducts are readily calculated. These absorption spectra are shown in Figure 4e. For irradiation times below 120 minutes, the absorption spectra of the photoproducts are very similar in general shape, changing only in intensity in a manner consistent with a higher photoproduct concentration over time (n.b. the NMR spectra also changed more profoundly after 120 minutes of irradiation time - see Figs. 5 and S6-S12). In contrast, after 120 minutes, the absorption bands of the photoproducts become less well resolved and begin to merge. This could indicate that initial irradiation produces a particular set of photoproducts that become different photoproducts as irradiation time lengthens, with a possibility of them being photoproducts of the photoproducts. This increase in the diversity of photoproducts (each with their own absorption signatures) would explain the increasing diversity of peaks in the NMR spectra and the smoothing in the absorption spectra with time. In any case, it is worth noticing that the shape of the absorption spectrum of the photoproducts is similar to that of anti- $\mathrm{B}_{18} \mathrm{H}_{22}$. This could mean that the chromophoric unit of the photoproducts is structurally similar to anti- $\mathrm{B}_{18} \mathrm{H}_{22}$ on which more will be said in the following section.

All the above observations clearly demonstrate that anti- $\mathrm{B}_{18} \mathrm{H}_{22}$ undergoes photochemical reactions upon high intensity irradiation. Nevertheless, they do not necessarily link this photodegradation to the promotion of anti- $\mathrm{B}_{18} \mathrm{H}_{22}$ to upper excited states. To establish whether the two are indeed connected, we performed an additional experiment of irradiating a sample of $1 \mathrm{mM} c$-hexane solution of anti- $\mathrm{B}_{18} \mathrm{H}_{22}$ for 150 minutes using the same conditions and experimental set-up as with the previous samples, but with an excitation pump laser with only a tenth of the intensity of previous runs, at $2.4 \mathrm{MW} \mathrm{cm}^{-2}$. Under these weaker pumping conditions, the percentage of absorbed photons that goes into ESA $\rho_{E S A}$ is importantly reduced (Figure 2). If the photodegradation were related to the total amount of absorbed energy, the sample irradiated for 150 mins with $2.4 \mathrm{MW} \mathrm{cm}^{-2}$ pulses should render the same or similar results as the sample irradiated for 15 mins with $24 \mathrm{MW} \mathrm{cm}{ }^{-2}$ pulses. In contrast, if the photodegradation were mediated by the promotion of anti- $\mathrm{B}_{18} \mathrm{H}_{22}$ to upper excited states, the low intensity irradiated sample should be significantly less photodegraded than the high intensity irradiated one. The absorption and differential absorption spectra of the sample irradiated for 150 mins (red dots in Figure 4a and 4b), as well as its ${ }^{11} \mathrm{~B}$ NMR spectrum (Fig. S12), show little to no photodegradation at all, indicating that the excitation of anti- $\mathrm{B}_{18} \mathrm{H}_{22}$ to upper excited states is fundamental to activate chemically reactive deexcitation pathways.

\subsection{Identification of anti-B ${ }_{18} \mathrm{H}_{22}$ photoproducts}

Thus far, we have established that upon high intensity irradiation anti- $\mathrm{B}_{18} \mathrm{H}_{22}$ participates in efficient ESA and the promotion to upper excited states in which the formation of photoproducts occurs. Identification of the exact nature of these photoproducts is however made very difficult by virtue of the high acidity of anti$\mathrm{B}_{18} \mathrm{H}_{22}$ and the new photoderivative species, and, therefore, their disinclination to separation and isolation using chromatographic techniques (n.b. various silica and alumina stationary phases and acidified solvent mobile phases were tried, none of which led to satisfactory separation). This is further complicated by the very small sample sizes available for separation work. Therefore, definitive structural characterisation of 
individual photoproducts was not achieved, but the use of NMR spectroscopy and, in particular, mass spectrometry, augmented by theoretical calculations, has enabled us to provide a likely composition of the photoproducts that are formed upon prolonged high intensity laser irradiation.

After 210 minutes of intense $355 \mathrm{~nm}$ UV irradiation, during which time 1.15 MJ of energy was delivered into the $c$-hexane sample of anti- $\mathrm{B}_{18} \mathrm{H}_{22}$, significant photodegradation of the borane occurred as evidenced in the UV-vis spectra presented in Figure 4 and in its ${ }^{11} \mathrm{~B}$ NMR spectrum (Figs. 5 and S11). From a borane chemist's perspective however, a more apt description would be photoinduced transformation rather than photodegradation, as the ${ }^{11} \mathrm{~B}$ NMR spectrum does not show a break-down or degradation of the 18 -vertex borane cluster molecule per se to, for example, monoboron boric acid, but rather the change into one or more related macropolyhedral borane species. As the absorption spectra of these new species (Figure 4) are very similar to that of anti- $\mathrm{B}_{18} \mathrm{H}_{22}$ it makes sense that the photoproducts are structurally related and that the photochemistry associated with the upper excited states of anti- $\mathrm{B}_{18} \mathrm{H}_{22}$ is subtle rather than destructive. Valuable insight into the composition of the photoproducts was achieved using mass spectrometry. Figure 6 shows the mass spectrum for the 210mins(1.15 MJ) sample (see S11 for its NMR and S13 for full mass spectrum) and a breakdown of this spectrum into simulated envelopes of its major components. 


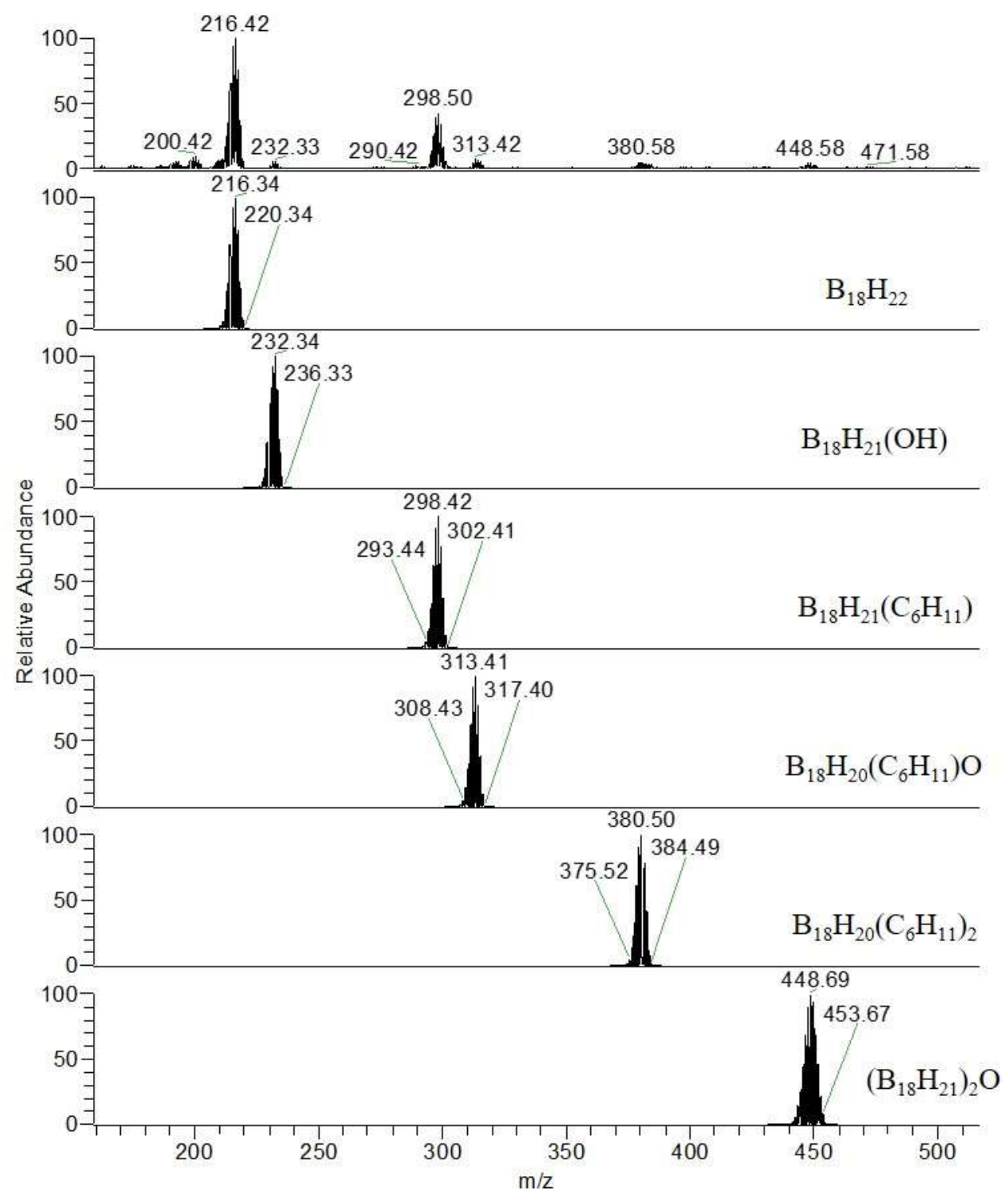

Figure 6: Electrospray Mass spectrum for the $210 \operatorname{mins}(1.15 \mathrm{MJ})$ sample of anti- $\mathrm{B}_{18} \mathrm{H}_{22}$ (top), with simulated mass envelopes for individual components underneath. See Methods and S13 for full description.

As boron naturally occurs in an isotopic ratio of ${ }^{11} \mathrm{~B}:{ }^{10} \mathrm{~B}$ of $80: 20$, then its compounds typically show the sort of isotope "envelopes" seen in Figure 6. The mass numbers $(\mathrm{m} / \mathrm{z})$ shown above these envelopes are for the mean atomic mass of boron (occasionally mass "cut off" values are also shown for ${ }^{11} \mathrm{~B}$ calculations). The spectrum shows a main peak at $\mathrm{m} / \mathrm{z} 216.42$ that corresponds exactly to the anion of $\mathrm{B}_{18} \mathrm{H}_{22}$. Additionally, there are several other peaks, the combined relative abundance of which is roughly equivalent to the that for anti- $\mathrm{B}_{18} \mathrm{H}_{22}$. This ratio of relative abundances between anti- $\mathrm{B}_{18} \mathrm{H}_{22}$ and its photoproducts is incidentally the same as that inferred from a comparison of integrations of resonance peaks in the ${ }^{11} \mathrm{~B}$ NMR spectrum mentioned in the discussion above. By far the most relevant mass peak amongst the photoproducts is at $\mathrm{m} / \mathrm{z}$ 298.42, which would match exactly to the mass of the anion of the $c$-hexanyl monosubstituted derivative of anti- $\mathrm{B}_{18} \mathrm{H}_{22}, \mathrm{~B}_{18} \mathrm{H}_{21}\left(\mathrm{C}_{6} \mathrm{H}_{11}\right)$, conceivably generated from light-induced reaction between molecules of anti- 
$\mathrm{B}_{18} \mathrm{H}_{22}$ in their upper excited state with surrounding solvent molecules of $c$-hexane. Two mono-substituted derivatives of anti- $\mathrm{B}_{18} \mathrm{H}_{22}, 7-\mathrm{I}-\mathrm{B}_{18} \mathrm{H}_{21}$ and 4- $\mathrm{Br}-\mathrm{B}_{18} \mathrm{H}_{21}$ are known ${ }^{6 a, 5}$ (the numbers 7 and 4 denote the position of substitution - see Figure 7 left), but neither have a ${ }^{11} \mathrm{~B}$ NMR spectrum with similar chemical shifts as those seen in Fig. S11 for the 210mins(1.15 MJ) sample. This suggests that the location of the $c$-hexanyl substituent on the $\left\{\mathrm{B}_{18}\right\}$-cluster is different to that of the known monosubstituted species. This, however, may be expected as 7-I- $\mathrm{B}_{18} \mathrm{H}_{21}$ nor $4-\mathrm{Br}-\mathrm{B}_{18} \mathrm{H}_{21}$ were synthesized by very different routes. It may be noted that the UV photolysis of deaerated $c$-hexane solutions of nido-decaborane-14, (anti- $\mathrm{B}_{18} \mathrm{H}_{22}$ is composed of two decaborane clusters sharing a common edge, see Figure 7) is known to produce cyclohexyl-nido-decaborane, predominantly 5cyclohexyl-nido-decaborane, in yields as high as $33 \%{ }^{37}$
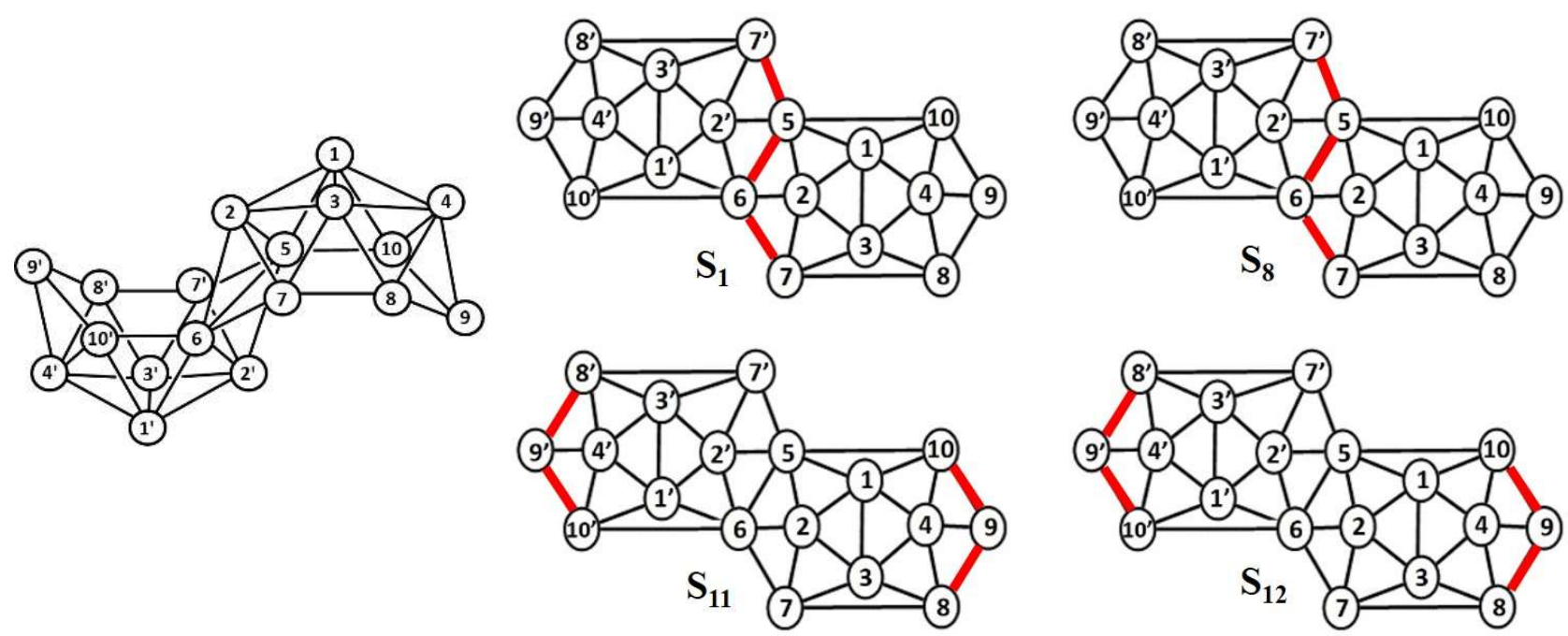

Figure 7. Numbering system for anti- $\mathrm{B}_{18} \mathrm{H}_{22}$ (left), and B-B connectivities with anti-bonding character (thick red lines) in the anti- $\mathrm{B}_{18} \mathrm{H}_{22}$ cluster found at its $\mathrm{S}_{1}, \mathrm{~S}_{8}, \mathrm{~S}_{11}$, and $\mathrm{S}_{12}$ excited states (right).

A clue to the possible location of the $c$-hexanyl substituent may be in the CASSCF calculated natural orbitals shown in Figure S14, which reveal the B-B connectivities in the 18-vertex cluster that incur antibonding character at the $S_{1}, S_{8}, S_{11}$, and $S_{12}$ excited states that are calculated to be the most probable to be populated on the basis of their respective oscillator strengths (see Table S1). Figure 7 (right) illustrates these connectivities with thick red lines. Upon population of these states, the highlighted B-B connectivities will tend to stretch due to their anti-bonding character and it is likely that they are the most probable site of reaction. As we can be sure from the UV-vis and NMR spectroscopy, as well as the mass spectrometry, that the 18vertex borane skeleton remains intact, then substitution at the B5 or B6 position, which conjoin the two subclusters, may be disregarded. Also, the dissimilarity of the ${ }^{11} \mathrm{~B}$ NMR with known derivative $7-\mathrm{I}_{-} \mathrm{B}_{18} \mathrm{H}_{21}$, ${ }^{6 a}$ suggests that substitution at the B7 (or symmetrically equivalent B7') position is also unlikely. Therefore, it is reasonable that the most probable position of the $c$-hexanyl substituent in $\mathrm{B}_{18} \mathrm{H}_{21}\left(\mathrm{C}_{6} \mathrm{H}_{11}\right)$ is at either the $\mathrm{B} 8$, $\mathrm{B} 9$, or $\mathrm{B} 10$ position, and that its formation occurs if a molecule of anti- $\mathrm{B}_{18} \mathrm{H}_{22}$ reaches its $\mathrm{S}_{11}$ or $\mathrm{S}_{12}$ state.

The mass peak at $\mathrm{m} / \mathrm{z} 380.50$ would correlate with the molecular mass of the anion of a disubstituted derivative $\mathrm{B}_{18} \mathrm{H}_{20}\left(\mathrm{C}_{6} \mathrm{H}_{11}\right)_{2}$, and the peak at $\mathrm{m} / \mathrm{z} 448.69$ would correlate with a $\left\{\mathrm{B}_{18}\right\}$-cluster dimer conjoined 
by an atom of oxygen: $\left(\mathrm{B}_{18} \mathrm{H}_{21}\right)_{2} \mathrm{O}$, but this is now no more than reasonable speculation, and, in the absence of more evidence, would require further investigation. Interestingly, however, if $c$-hexane solutions are irradiated for an even longer period of 270 minutes, then ${ }^{11} \mathrm{~B}$ NMR (see Figure S15) and mass spectrometry (see Figure S16) show a further diminishing in the relative abundance of anti- $\mathrm{B}_{18} \mathrm{H}_{22}$, but also in the relative abundances of $\mathrm{B}_{18} \mathrm{H}_{21}\left(\mathrm{C}_{6} \mathrm{H}_{11}\right)$ and $\mathrm{B}_{18} \mathrm{H}_{20}\left(\mathrm{C}_{6} \mathrm{H}_{11}\right)_{2}$, and a substantial increase in the relative abundance of what we are tentatively characterizing as $\left(\mathrm{B}_{18} \mathrm{H}_{21}\right)_{2} \mathrm{O}$, suggesting that the formation of the $c$-hexanyl substituted cluster might precede the emergence of the $\left\{\mathrm{B}_{18}\right\}_{2} \mathrm{O}$ dimer. As a last note, the high concentration of $\left(\mathrm{B}_{18} \mathrm{H}_{21}\right)_{2} \mathrm{O}$ visibly quenches much of the luminescence of the sample (see Figure S17). A more rigorous exclusion of air from the system would likely eliminate oxygenated products. ${ }^{37}$

\section{Conclusions}

In summary, the amplification properties of any laser material are essentially determined by the effective

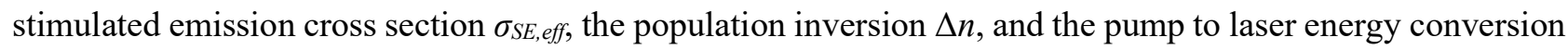
$\eta_{p}$ (see Equation 1). The higher these values are, the better the laser performance becomes. In this work we have shown that for the particular case of anti- $\mathrm{B}_{18} \mathrm{H}_{22}$, (i) the presence of excited state absorption (ESA) at the emission region strongly reduces the maximum $\sigma_{S E \text {,eff, }}$ (ii) effective singlet-singlet annihilation, when solutions are pumped above threshold, reduces the available $\Delta n$, and (iii) the ESA at the pump wavelength strongly diminishes $\eta_{p}$. All these effects, together with the observed reduction in luminescence quantum yield due to concentration quenching and the increase in scattering losses due to solubility problems, ${ }^{7}$ contribute to severely reducing the laser efficiency and fully account for the surprising underperformance of anti- $\mathrm{B}_{18} \mathrm{H}_{22}$ as a laser material. It is only by virtue of its exceptional emission properties (quantum yield near unity and nanosecond lifetime) that anti- $\mathrm{B}_{18} \mathrm{H}_{22}$ manages to partially compensate its many drawbacks.

In addition, by means of UV-vis spectroscopy, NMR spectroscopy, as well as Mass spectrometry, we have revealed that the population of upper excited states upon sequential absorption of two $355 \mathrm{~nm}$ photons results in the activation of chemically reactive relaxation pathways. This, in turn, leads to the formation of photochemically produced novel molecular species. In particular, the most likely and abundant photoproduct formed upon prolonged high intensity laser irradiation is a $c$-hexanyl substituted derivative of anti- $\mathrm{B}_{18} \mathrm{H}_{22}$.

In conclusion, this study suggests that the current absence of new fluorescence boranes displaying laser emission could be indicative that the presence of ESA losses is inherent to the existing boranes and represents their Achilles' heel. In this sense, anti- $\mathrm{B}_{18} \mathrm{H}_{22}$ could be the exception rather than the rule. However, it is entirely possible that these limiting factors in the photophysical properties of the excited states of anti- $\mathrm{B}_{18} \mathrm{H}_{22}$ may be advantageously modified by the introduction of substituents on to the octadecaborane cluster and we hope to address this potential in forthcoming publications. 


\section{Methods:}

Sample preparation and handling: anti- $\mathrm{B}_{18} \mathrm{H}_{22}$ was synthesized as described elsewhere. ${ }^{38}$ It was dissolved in $c$-hexane that had been dried over $\mathrm{CaH}_{2}$ and distilled, and diluted to the required concentration for each experiment and stirred overnight until complete dissolution. For the measurement of the ESA cross section at the pump wavelength, solutions of $0.5 \mathrm{mM}$ where prepared. For the long irradiation experiment, glass vials were filled with $6 \mathrm{~mL}$ of $1 \mathrm{mM}$ solution. Small aliquots $(0.2 \mathrm{~mL})$ of the irradiated solutions were extracted to perform photophysical measurements. Dichloromethane (DCM) was added to the aliquots (c-hex:DCM ratio 2:1) to dissolve the white precipitate generated during irradiation. This way, the deleterious effects of the light scattering induced by the white precipitate were avoided. Regarding the NMR study, each sample, for purposes of thoroughness, was carefully opened, a small aliquot of the original $c$-hexane solution was subsequently taken and its ${ }^{11} \mathrm{~B}$ NMR spectrum measured. Once these initial experiments were done, the samples were evaporated to dryness, and re-dissolved in fresh deuterated-dichloromethane, $\mathrm{CD}_{2} \mathrm{Cl}_{2}$, and their ${ }^{11} \mathrm{~B}$ and ${ }^{1} \mathrm{H}$ NMR spectra measured. The reason for both $c$-hexane and $\mathrm{CD}_{2} \mathrm{Cl}_{2}$ measurements was to be doubly sure that the change in solvent had no effect on the samples being measured, which indeed was the case, and as such, only $\mathrm{CD}_{2} \mathrm{Cl}_{2}$ solutions of the samples are shown. For the Mass spectrometric experiments, all samples were evaporated and redissolved in acetonitrile.

Transient absorption measurements: Prior to transient absorption measurements, the anti- $\mathrm{B}_{18} \mathrm{H}_{22}$ sample was diluted in $n$-hexane to a concentration of OD 1.7 at the pump wavelength ( $340 \mathrm{~nm}$ ) in a $2 \mathrm{~mm}$ path length quartz cuvette. A magnetic stir bar, placed inside the cuvette, was rotated during whole measurement to prevent/reduce photo-degradation. For the time-resolved transient absorption measurements, the femtosecond pulses were generated by a modular laser system consisting of a Ti:sapphire regenerative amplifier (Spitfire Ace-100F, Spectra-Physics, USA) seeded with a Ti:sapphire oscillator (MaiTai SP, Spectra-Physics), and pumped by Nd:YLF laser (Empower 30, Spectra-Physics). The laser system produces $\sim 100 \mathrm{fs}$ pulses centred at $800 \mathrm{~nm}$ with a $1-\mathrm{kHz}$ repetition rate. The output was divided into excitation and probe beams by a beam splitter. The excitation beam was transformed by an optical parametric amplifier (TOPAS Prime, Light Conversion, Lithuania) to final wavelength of $340 \mathrm{~nm}$. The probe pulses were generated by focusing the beam on a $3 \mathrm{~mm} \mathrm{CaF}_{2}$ plate, which was continuously moving. This setup allowed to generate a white light continuum covering the 350-700 nm spectral region. All the reflective optics which guided the white-light beam were covered by UV enhanced aluminum to minimize losses in UV spectral region and to minimize chirp and chromatic aberration, the beam was collimated by an off-axis parabolic mirror and split by a broadband, 50/50 beam splitter to a reference and probe beam. The probe beam was focused by a $250 \mathrm{~mm}$ spherical mirror to the sample where it overlapped with the excitation beam. Probe and reference beams were dispersed by a grating in Czerny-Turner monochromator and detected by a pair of CCD arrays. The pump polarization was set at a magic angle $\left(54.7^{\circ}\right)$ relative to the probe polarization to eliminate any effects from rotational diffusion. The pump photon density at the sample was kept below $10^{14}$ photon. $\mathrm{cm}^{-2}$.pulse $\mathrm{e}^{-1}$. The transient absorption 
spectra were chirp corrected (software CarpetView, Light Conversion, Lithuania) by manually choosing the time zero for more than ten wavelength points and fitting it to a polynomial equation.

Laser irradiation set-up: The experimental set-ups can be seen in Figure S2. The vials or quartz cuvettes containing the anti- $\mathrm{B}_{18} \mathrm{H}_{22} / c$-hexane solutions were optically pumped at $355 \mathrm{~nm}$ with laser pulses from the third harmonic of Neodymium $\left(\mathrm{Nd}^{3+}\right)$ based lasers: for the intensity dependent transmission measurements a Nd:YVO 4 laser (Lotis TII SL-2132, 7 ns FWHM pulses, $15 \mathrm{~Hz}$ repetition rate), and for the long irradiation experiment a Nd:YAG laser (Spectron SL282G, 7 ns FWHM pulses, $10 \mathrm{~Hz}$ repetition rate) were used. Attenuators and a cut-off filter were used, respectively, to control the pump energy and remove residual 532 $\mathrm{nm}$ light from the second harmonic. The laser radiation was focused onto the air/solution interface with a spherical lens of $50 \mathrm{~cm}$ focal length (spot size, $3 \mathrm{~mm}$ in diameter). A prism was used to send the radiation downwards. This pump configuration was used to avoid irradiating at the interphase between quartz and solvent, as we detected the appearance of some sort of interaction or laser ablation leading to the formation of bubbles and the deposition of white markings in the cuvette wall. ${ }^{7}$ The incident and transmitted energies were measured with either pyroelectric (GenTec 12LP-S) or thermopile (Newport 818p-030-18hp) energy meters.

Steady-state photophysical measurements: the absorption spectra were recorded in an Analytik Jena SPECORD 600 spectrophotometer. The fluorescence and excitation spectra were acquired with the spectrofluorimeter HORIBA Jobin Yvon Fluoromax-4. The emission spectra were collected in front-face configuration (excitation at $30^{\circ}$, detection at $60^{\circ}$ ). Quartz cuvettes with a $1 \mathrm{~mm}$ path-length were used for both absorbance and emission measurements.

Quantum chemical computations: The Franck-Condon $\left(\mathrm{S}_{0} \mathrm{~min}\right)$ and $\mathrm{S}_{1}$ minimum geometries of anti- $\mathrm{B}_{18} \mathrm{H}_{22}$ were taken from a previous work. ${ }^{3}$ GSA computations were conducted on top of the $\mathrm{S}_{0}$ equilibrium geometry by calculating $N_{\text {tot }}=8$ states in the state-average (SA)-CASSCF procedure. Meanwhile, ESA and SE computation were carried out on top of the $\mathrm{S}_{1}$ equilibrium geometry by using a SA-CASSCF wavefunction with $N_{\text {tot }}=20$ states to obtain enough high-energy excited states to study the corresponding transition bands. The wave functions were built distributing 12 electrons into 12 molecular orbitals since this active space has been proven adequate in the description of excited states of anti- $\mathrm{B}_{18} \mathrm{H}_{22}$ and related borane systems. ${ }^{4,6,39,40}$ Electronic dynamic correlation was computed by means of the state-specific CASPT2 method or its multistate(MS)-CASPT2 variant giving, overall, similar results. An imaginary level shift of 0.2 a.u. was used to minimize the effect of weakly interacting intruder states, whereas the ionization-potential electron-affinity (IPEA) parameter was set to the value of 0.25 a.u., ${ }^{41}$ in coherence with previous works on related systems. 4,6,39,40 Oscillator strengths between an initial $(i)$ and final $(f)$ electronic states $\left(f_{i f}\right)$ were computed using the SA-CASSCF wave functions $\left(\Psi_{\mathrm{i}}, \Psi_{f}\right)$ and the CASPT2 (or MS-CASPT2) vertical energies $\left(v_{i f}\right)$, according to the formula $f_{i f}=\frac{2 m v_{i f}}{3 \hbar^{2} e^{2}} M_{i f}^{2}$, where $m$ and $e$ are the electron mass and charge, respectively, $M_{i f}$ stands for the 
transition dipole moment $M_{i f}=\left\langle\Psi_{i}\left|\mu_{e}\right| \Psi_{f}\right\rangle$, being $\mu_{e}$ the electric dipole moment, as described in detail elsewhere. ${ }^{42}$ All multiconfigurational calculations were conducted using the double- $\zeta$ ANO-S-VDZP basis set, without imposing symmetry restrictions, and employing the MOLCAS 8 software package. ${ }^{43}$

NMR and Mass spec set-ups: The NMR study was made using a Varian $400 \mathrm{MHz}$ instrument. Mass spectrometric measurements were made on a LC Mass Spectrometry ThermoScientific LCQ Fleet Ion Trap device. All samples were submitted to the following measurement conditions: Source Voltage of $4.98 \mathrm{kV}$; Source Current of $1.90 \mu \mathrm{A}$; Sheath Gas Flow Rate of 15units; Capillary Voltage of $-14.04 \mathrm{~V}$; Capillary Temperature of $275^{\circ} \mathrm{C}$; Flow Rate of $15 \mu \mathrm{L} / \mathrm{min}$.

Calculation of cross sections (experiment): The Ground State Absorption (GSA) cross section was calculated with the expression $\sigma_{\mathrm{GSA}}\left(\mathrm{cm}^{2}\right)=\ln (10) \cdot 1000 \cdot \varepsilon / \mathrm{N}_{\mathrm{A}}$, where $\varepsilon$ is the molar extinction coefficient (in $\left.\mathrm{M}^{-1} \mathrm{~cm}^{-1}\right)$, and $\mathrm{N}_{\mathrm{A}}$ is Avogadro's number. The stimulated emission (SE) cross-section is given by $\sigma_{\mathrm{SE}}(\lambda)=\lambda^{4} \mathrm{E}(\lambda) /\left(8 \pi c n^{2} \tau\right)$, where $\mathrm{E}(\lambda)$ is the $\mathrm{S}_{1}-\mathrm{S}_{0}$ fluorescence spectrum normalized, so that $\int \mathrm{E}(\lambda) \mathrm{d} \lambda=\phi, \phi$ being the fluorescence quantum yield, $c$ is the speed of light in vacuum, $n$ is the refractive index of the solution (1.43), and $\tau$ is the fluorescence life-time.

Calculation of cross sections (computational): It can be shown (see Supplementary Information, Section S2) that the absorption cross-section spectra (in $\mathrm{cm}^{2}$ ), both for GSA and ESA, can be approximated as:

$$
\sigma(v)=\sum_{j} 1.03 \times 10^{-16} \frac{f_{i j}}{F\left(n, v_{i j}\right) \Delta v} e^{-\frac{\left(v-v_{i j}\right)^{2}}{2(\Delta v / 2.3548)^{2}}}
$$

where $i$ and $f$ stand for all the electronic transitions between the initial and final electronic states, respectively, contributing to the spectrum (for GSA, $i=0$ and $f=1 \ldots N$; for ESA, $i=1$ and $f=2 \ldots N$ ). $f_{i f}$ and $v_{i f}$ are the oscillator strength and vertical energy (in $\mathrm{eV}$ ) of transition $i \rightarrow f$, respectively, $\Delta v$ is the transition full width half maximum (FWHM), and $F\left(n, v_{i f}\right)$ is the local field factor that takes the solvent effects into account. In the Onsager real cavity approximation, ${ }^{44}$ it is given by $F\left(n, v_{i f}\right)=\left(2 n\left(v_{i f}\right)^{2}+1\right)^{2} /\left(9 n\left(v_{i f}\right)^{3}\right)$, with $n\left(v_{i f}\right)$ being the solvent refractive index at transition energy $v_{i f}$. A FWHM $\Delta v=0.45 \mathrm{eV}$ is seen to reproduce at best the experimental results for GSA and ESA.

The stimulated emission cross section according to the Einstein coefficients is given by:

$$
\sigma_{S E}(v)=A_{1 \rightarrow 0} \frac{h^{3} c^{2}}{8 \pi n^{2} v^{2}} g(v)
$$

where $\mathrm{A}_{1 \rightarrow 0}$ is the radiative decay rate, $h$ is the Planck constant, $c$ the speed of light in vacuum, $n$ the refractive index, and $g(v)$ is the fluorescence spectral line-shape normalized so that $\int \mathrm{g}(v) \mathrm{d} v=1$. In this case it is assumed to be a Gaussian of FWHM $\Delta v=0.5 \mathrm{eV}$ and centred at the transition energy given by the computed $\mathrm{S}_{1}-\mathrm{S}_{0}$ transition energy (table S1). When dealing with molecules, the radiative decay rate is best calculated from the 
computed absorption cross sections $\sigma_{\mathrm{A}}(\lambda)$ in $\mathrm{nm}^{2}$ and the fluorescence line-shape $g(\lambda)$ making use of the Strickler-Berg formula: ${ }^{45}$

$$
A_{1 \rightarrow 0}=\tau_{\text {rad }}^{-1}=\frac{8 \pi c n_{F}^{3}}{n_{A}} \frac{\int_{e m} g(\lambda) d \lambda}{\int_{e m} g(\lambda) \lambda^{3} d \lambda} \int_{a b s} \frac{\sigma_{A}(\lambda)}{\lambda} d \lambda
$$

where $\mathrm{n}_{\mathrm{F}}$ and $\mathrm{n}_{\mathrm{A}}$ are the average refractive indices of the solution in the fluorescence and absorption regions, respectively, and $c$ is the speed of light in vacuum (in units of $\mathrm{nm} / \mathrm{ns}$ ). The emission integrals extend over the region of $\mathrm{S}_{1}-\mathrm{S}_{0}$ emission $(\mathrm{em})$ and the absorption integral extends over the main band of the $\mathrm{S}_{0}-\mathrm{S}_{1}$ absorption (abs).

Calculation of Förster Radius for Singlet-Singlet Annihilation (SSA): The Förster radius $R_{0}$ is defined as the donor-acceptor (excited molecules) separation for which the donor emits and transfers (to the acceptor) its energy with the same probability. For SSA, $R_{0}$ depends on the excited state photophysical properties of the excited species and is given by ${ }^{46}$

$$
R_{0}{ }^{6}=\frac{9000(\ln 10) \kappa^{2} \phi_{f}}{128 \pi^{5} N_{A} n^{4}} \int_{0}^{\infty} g(\lambda) \varepsilon_{E S A}(\lambda) \lambda^{4} d \lambda
$$

In Eq. (3) the integral accounts for the spectral overlap of the fluorescence spectrum $g(\lambda)\left(\int g(\lambda) \mathrm{d} \lambda=1\right)$ with the ESA spectrum of the acceptor $\varepsilon_{E S A}(\lambda)\left(\mathrm{M}^{-1} \mathrm{~cm}^{-1}\right)$. The wavelength $\lambda$ is expressed in units of $\mathrm{cm}$. $\phi_{f}$ and $n$ are the fluorescence quantum yield of the excited molecule in the absence of other excited molecules and the refractive index of the host medium, respectively, and $\kappa^{2}$ is the orientation factor which accounts for the relative orientation of excited molecules transition dipole moments in the host medium. In the quantum chemical computations $g(\lambda)$ and $\varepsilon_{E S A}(\lambda)$ were computed (see above). $\phi_{f}$ was taken as $0.97,{ }^{3} n \sim 1.44$ (c-hexane) and $\kappa^{2} \sim 2 / 3$ (isotropic dynamic averaging). Substituting these values into eq. (3), leads to Förster radii of $R_{0}=$ $4 \mathrm{~nm}(\mathrm{CASPT} 2)$ and $R_{0}=4.2 \mathrm{~nm}(\mathrm{MS}-\mathrm{CASPT} 2)$.

Calculation of molar fraction of surviving anti- $B_{18} H_{22}$ molecules: The absorption spectrum of the photoproducts at each irradiation time $\left(\mathrm{A}_{\mathrm{pp}}\left(t_{i r r}\right)\right)$ can be calculated as $\mathrm{A}_{\mathrm{pp}}\left(t_{i r r}\right)=\mathrm{A}\left(t_{i r r}\right)-\mathrm{X}_{\mathrm{m}}\left(t_{i r r}\right) \cdot \mathrm{A}(0)$, where $\mathrm{A}\left(t_{i r r}\right)$ is the measured absorbance of the irradiated sample, $\mathrm{X}_{\mathrm{m}}\left(t_{i r}\right)$ is the molar fraction of surviving anti$\mathrm{B}_{18} \mathrm{H}_{22}$, and $\mathrm{A}(0)$ is the absorbance of the non-irradiated solution (reference sample 0mins). As it is likely that the only emissive species in the irradiated solution is anti- $\mathrm{B}_{18} \mathrm{H}_{22}$, then we are able to infer a reasonable approximation of the molar fraction from the changes in the excitation spectra with time. In solutions where there are multiple absorbing species but only one emissive species, the excitation spectrum $F\left(t_{\text {irr }}\right)$ (for low absorbance and negligible reabsorption) is approximately given by $F\left(t_{i r r}\right) \approx \mathrm{f} \cdot \mathrm{X}_{\mathrm{m}}\left(t_{i r r}\right) \cdot \mathrm{A}(0) \cdot{ }^{47} \mathrm{f}$ is a proportionality factor containing information on the fluorescent compound emission properties and the apparatus configuration, and $\mathrm{X}_{\mathrm{m}}\left(t_{\text {irr }}\right)$ and $\mathrm{A}(0)$ are, respectively, the molar fraction of surviving anti- $\mathrm{B}_{18} \mathrm{H}_{22}$ and the absorbance spectrum of the non-irradiated solution (reference sample). Obviously, $\mathrm{X}_{\mathrm{m}}(0)=1$. Thus, it 
is easy to see that the molar fraction of surviving anti- $\mathrm{B}_{18} \mathrm{H}_{22}$ molecules in the irradiated samples can be calculated as $\mathrm{X}_{\mathrm{m}}\left(t_{i r r}\right)=F\left(t_{i r r}\right) / F(0)$.

\section{References}

1. (a) "Boron Science: New Technologies and Applications", $1^{\text {st }}$ Edition, Ed. Narayan S. Hosmane, CRC Press (2012); (b) "Handbook of Boron Science", Ed. Narayan S. Hosmane and Robert eagling, World Scientific (2018).

2. (a) S. Mukherjee, P. Thilagar, Boron clusters in luminescent materials, Chem. Commun., 2016, 52, 10701093; (b) R. Núñez, M. Tarrés, A. Ferrer-Ugalde, F. Fabrizi de Biani, F. Teixidor, Electrochemistry and photoluminescence of icosahedral boranes, metallacarboranes, and their derivatives, Chem. Rev., 2016, $116,23,14307-14378$.

3. Londesborough, M. G. S.; Hnyk, D.; Bould, J.; Serrano-Andrés, L.; Sauri, V.; Oliva, J. M.; Kubat, P.; Polivka, T.; Lang, K. Distinct Photophysics of the Isomers of $\mathrm{B}_{18} \mathrm{H}_{22}$ Explained. Inorg. Chem. 2012, 51 (3), 1471-1479.

4. (a) Londesborough, M. G. S.; Dolanský, J.; Cerdán, L.; Lang, K.; Jelínek, T.; Oliva, J. M.; Hnyk, D.; Roca-Sanjuán, D.; Francés-Monerris, A.; Martinčík, J.; Nikl, M.; Kennedy, J. D. Thermochromic Fluorescence from $\mathrm{B}_{18} \mathrm{H}_{20}\left(\mathrm{NC}_{5} \mathrm{H}_{5}\right)_{2}$ : An Inorganic-Organic Composite Luminescent Compound with an Unusual Molecular Geometry. Adv Opt Mater 2017, 5 (6), 1600694; (b) Londesborough, M. G. S.; Dolanský, J.; Jelínek, T.; Kennedy, J. D.; Císařová, I.; Kennedy, R. D.; Roca-Sanjuán, D.; FrancésMonerris, A.; Lang, K.; Clegg, W. Substitution of the laser borane anti- $\mathrm{B}_{18} \mathrm{H}_{22}$ with pyridine: a structural and photophysical study of some unusually structured macropolyhedral boron hydrides. Dalton Trans. 2018, 47 (5), 1709-1725.

5. Spokoyny, A.; Anderson, K. P..; Waddington, M. A.; Balaich, G. J.; StauberJ.; Caram J. R.; Djurovich P.I.; A Molecular Boron Cluster-Based Chromophore with Dual Emission. Dalton Trans. 2020,

6. (a) Londesborough, M. G. S.; Dolanský, J.; Bould, J.; Braborec, J.; Kirakci, K.; Lang, K.; Císařová, I.; Kubát, P.; Roca-Sanjuán, D.; Francés-Monerris, A.; Slušná, L.; Noskovičová, E.; Lorenc, D. Effect of Iodination on the Photophysics of the Laser Borane anti- $\mathrm{B}_{18} \mathrm{H}_{22}$ : Generation of Efficient Photosensitizers of Oxygen. Inorg. Chem. 2019, 58 (15), 10248-10259; (b) Sauri, V.; Oliva, J. M.; Hnyk, D.; Bould, J.; Braborec, J.; Merchan, M.; Kubat, P.; Císařová, I.; Lang, K.; Londesborough, M. G. S. Tuning the Photophysical Properties of anti- $\mathrm{B}_{18} \mathrm{H}_{22}$ : Efficient Intersystem Crossing between Excited Singlet and Triplet States in New 4,4'-(HS)2-anti-B ${ }_{18} \mathrm{H}_{20}$. Inorg. Chem. 2013, 52 (16), 9266-9274.

7. Cerdán, L.; Braborec, J.; Garcia-Moreno, I.; Costela, A.; Londesborough, M. G. S. A borane laser. Nat. Commun. 2015, 6, 5958 DOI: 10.1038/ncomms6958. 
8. (a) Tan, C.; Chen, J.; Zhang, L.; Zhang, B.; Huang, X.; Meng, H. The preparation and characterization of $n$ - $\mathrm{B}_{18} \mathrm{H}_{22}$-beta cyclodextrin inclusion complex. IOP Conference Series: Earth and Environmental Science 2019, 233, 022005; (b) Zhang, B.; Chen, J.; Xiong, L.; Zhang, L.; Tan, C.; Meng, H. Hydrolysis Kinetic of New Laser Material $n-\mathrm{B}_{18} \mathrm{H}_{22}$. IOP Conference Series: Earth and Environmental Science 2019, 242 , 022040 .

9. Londesborough, M. G. S.; Lang, K.; Clegg, W.; Waddell, P. G.; Bould, J. Swollen Polyhedral Volume of the anti- $\mathrm{B}_{18} \mathrm{H}_{22}$ Cluster via Extensive Methylation: anti- $\mathrm{B}_{18} \mathrm{H}_{8} \mathrm{Cl}_{2} \mathrm{Me}_{12}$. Inorg. Chem. 2020, 59 (5), 26512654.

10. (a) F. P. Schaefer, Dye Lasers $3^{\text {rd }}$ Ed. (Springer, 1990); (b) A. E. Siegman, Lasers (Univ. Sci. Book, 1986);

(c) W. Koechner, Solid-state laser engineering $6^{\text {th }}$ Ed. (Springer, 2006).

11. D. Roca-Sanjuán, F. Aquilante, R. Lindh, "Multiconfiguration second-order perturbation theory approach to strong electron correlation in chemistry and photochemistry", Wiley Interdisciplinary Reviews, Computational Molecular Science, 2, (4), 585-603.

12. E. Sahar and D. Treves, "Excited singlet-state absorption in dyes and their effect on dye lasers," $J$. Quantum Electron. QE-13, 962-967 (1977).

13. R. Berera, R. van Grondelle and J. T. Kennis, "Ultrafast transient absorption spectroscopy: principles and application to photosynthetic systems," Photosynth. Res. 101, 105-118 (2009).

14. J. J. Serrano-Andrés, L. Serrano-Pérez, Calculation of Excited States: Molecular Photophysics and Photochemistry on Display, Handbook of Computational Chemistry, 639-725.

15. W. Holzer, H. Gratz, T. Schmitt, A. Penzkofer, A. Costela, I. García-Moreno, R. Sastre, and F. J. Duarte, "Photo-physical characterization of rhodamine $6 \mathrm{G}$ in a 2-hydroxyethyl-methacrylate methylmethacrylate copolymer," Chem. Phys. 256, 125-136 (2000).

16. Y. Dong, D. Saini, L. A. Echegoyen, R. Podila, "Passive optical switches based on endohedral fullerenes," Opt. Mater. 53, 14-18 (2016).

17. W. Heudorfer, and G. Marowsky, "Threshold singularities of optically pumped dye lasers," Appl. Phys. 17, 181-187 (1978).

18. K. Dasgupta and L. G. Nair, "Effect of excited-state absorption at signal wavelength in pulsed-dye-laser amplifiers," J. Quantum Electron. 26, 189-192 (1990).

19. P. R. Hammond, "Spectra of the lowest excited singlet states of rhodamine $6 \mathrm{G}$ and rhodamine B," $J$. Quantum Electron. QE-15, 624-632 (1979).

20. F. Sanchez and A. Kellou, "Laser dynamics with excited-state absorption," J. Opt. Soc. Am. B 14, 209213 (1997).

21. A. Sennaroglu, "Classification of power-degrading mechanisms in an optically pumped four-level laser: an analytical approach," J. Opt. Soc. Am. B 36, 2202-2209 (2019).

22. K. Petermann, "The role of excited-state absorption in tunable solid-state lasers," Opt. Quant. Electron. 22, S199-S218 (1990).

23. S. Y. Arzhantsev, A. Y. Chikishev, N. I. Koroteev, S. A. Magnitskii, and V. V. Shubin, "On the singlet- 
singlet annihilation of the excited states of rhodamine 3B in a polymer film," Laser Physics 9, 466-469 (1999).

24. J. Hofkens, M. Cotlet, T. Vosch, P. Tinnefeld, K. D. Weston, C. Ego, A. Grimsdale, K. Müllen, D. Beljonne, J. L. Brédas, S. Jordens, G. Schweitzer, M. Sauer, and F. De Schryver, "Revealing competitive Förster-type resonance energy-transfer pathways in single bichromophoric molecules," PNAS 100, 13146$13151(2002)$

25. C. Gärtner, C. Karnutsch, U. Lemmer, and C. Pflumm, "The influence of annihilation processes on the threshold current density of organic laser diodes," J. Appl. Phys. 101, 023107 (2007)

26. M. A. Stevens, C. Silva, D. M. Russell, and R. H. Friend, "Exciton dissociation mechanisms in the polymeric semiconductors poly(9,9-dioctylfluorene) and poly(9,9-dioctylfluorene-co-benzothiadiazole)," Phys. Rev. B 63, 165213 (2001).

27. L. Cerdán, E. Enciso, V. Martín, J. Bañuelos, I. López-Arbeloa, A. Costela and I. García-Moreno, "FRETassisted laser emission in colloidal suspensions of dye-doped latex nanoparticles," Nat. Photonics 6, 621626 (2012).

28. S. Speiser and A. Bromberg, "Photoquenching III. Analysis of the dependence of pulsed-laser-pumped dye laser performance on pumping conditions and on the dye molecular characteristics," Chem. Phys. 9, 191-197 (1975).

29. M. L. Kliewer and R. C. Powell, "Excited State Absorption of Pump Radiation as a Loss Mechanism in Solid-state Lasers," J. Quantum Electon. 25, 1850-1854 (1989)

30. W. R. Kerridge-Johns and M. J. Damzen, "Analysis of pump excited state absorption and its impact on laser efficiency," Laser Phys. Lett. 12, 125002 (2015).

31. I. Wieder, "Quenching of laser dye fluorescence by absorption from an excited singlet state," Appl. Phys. Lett., 21, 318-320 (1972).

32. S. Speiser, R. Van der Werf, and J. Kommandeur, "Photoquenching: the dependence of the primary quantum yield of a monophotonic laser-induced photochemical process on the intensity and duration of the exciting pulse," Chem. Phys. 1, 297-305 (1973).

33. R. B. King, "Three-dimensional aromaticity in polyhedral boranes and related molecules," Chem. Rev. 101, 1119-1152 (2001)

34. J. C. Green, M. L. H. Green and G. Parkin, "The occurrence and representation of three-centre twoelectron bonds in covalent inorganic compounds," Chem. Comm. 48, 11481-11503 (2012).

35. F. J. Duarte (ed.), High-Power Dye Lasers (Springer, 1991).

36. Fontaine, X. L. R.; Greenwood, N. N.; Kennedy, J. D.; MacKinnon, P. Boron-11 and proton nuclear magnetic resonance study of anti- $\mathrm{B}_{18} \mathrm{H}_{22}$ and its anions, anti- $\left[\mathrm{B}_{18} \mathrm{H}_{21}\right]^{-}$and anti- $\left[\mathrm{B}_{18} \mathrm{H}_{20}\right]^{2-}$. The crystal and molecular structure of $\left[\mathrm{NMe}_{4}\right]_{2}\left[\right.$ anti- $\left.\mathrm{B}_{18} \mathrm{H}_{20}\right]$. J. Chem. Soc., Dalton Trans. 1988, (7), 1785-1793.

37. Boocock, S. K.; Cheek, Y. M.; Greenwood, N. N.; Kennedy, J. D. A new route to isomers of icosaborane(26), $\mathrm{B}_{20} \mathrm{H}_{26}$. The use of $115.5-\mathrm{MHz}{ }^{11} \mathrm{~B}$ and ${ }^{11} \mathrm{~B}-\left\{{ }^{1} \mathrm{H}\right\}$ nuclear magnetic resonance spectroscopy for the comparison and characterisation of separated isomers and the identification of three 
further icosaboranes as 1,2'-, 2,5'-, and 5,5'(or 5,7')-( $\left.\mathrm{B}_{10} \mathrm{H}_{13}\right)_{2}$. J. Chem. Soc., Dalton Trans. 1981, (6), 1430-143.

38. Y. Li, and L. G. Sneddon, "Improved synthetic route to $n-\mathrm{B}_{18} \mathrm{H}_{22}$," Inorg. Chem. 45, 470-471 (2006).

39. J. Macháček, A. Francés-Monerris, N. Karmodak, D. Roca-Sanjuán, J. Fanfrlík, M.G.S. Londesborough, D. Hnyk, E.D. Jemmis, "A theoretical analysis of the structure and properties of $\mathrm{B}_{26} \mathrm{H}_{30}$ isomers. Consequences to the laser and semiconductor doping capabilities of large borane clusters", Phys. Chem. Chem. Phys., 2019, 21, 12916-12923.

40. A. Francés-Monerris, J. Holub, D. Roca-Sanjuán, D. Hnyk, K. Lang and J. Oliva-Enrich, „Photochromic Systém among Boron Hydrides: The Hawthorne Rearrangement“, J. Phys. Chem. Lett., 2019, 10, 20, 6202-6207.

41. G. Ghigo, B.O. Roos, P. Malmqvist, „A modified definition of the zeroth-order Hamiltonian in multiconfigurational perturbation theory (CASPT2)“, Chem. Phys. Lett., 396, 1-3, 2004, 142-149.

42. (a) Francés-Monerris, A., Segarra-Martí, J., Merchán, M., D. Roca-Sanjuán, Theoretical study on the excited-state $\pi$-stacking versus intermolecular hydrogen-transfer processes in the guaninecytosine/cytosine trimer. Theor Chem Acc 135, 31 (2016); (b) R. C. Hilborn, Einstein coefficients, cross sections, fvalues, dipole moments, and all that. Amer. J. of Phys., 50, 982 (1982).

43. R. Lindh et al., "MOLCAS 8: New capabilities for multiconfigurational quantum chemical calculations across the periodic table", J. Comp. Chem., 37, 5, 506-541.

44. K. Dolgaleva and R. W. Boyd, "Local-field effects in nanostructured photonic materials," Adv. Opt. Photon. 4, 1-77 (2012).

45. (a) S. J. Strickler and R.A. Berg, "Relationship between Absorption Intensity and Fluorescence Lifetime of Molecules" J. Chem. Phys. 37, 814 (1962). (b) J. B. Birks and D.J. Dyson, “The relations between the fluorescence and absorption properties of organic molecules" Proc. Roy. Soc. London A 275, 135 (1963).

46. M. A. Stevens , C. Silva, D. M. Russel, and R. H. Friend, "Exciton dissociation mechanisms in the polymeric semiconductors poly(9,9-dioctylfluorene) and pol(9,9-dioctylfluorene-co-benzothiadiazol)," Phys. Rev. B 63, 165213 (2001)

47. I. Lopez-Arbeloa, "Fluorescence quantum yield evaluation: corrections for re-absorption and reemission," J. Photochem. 14, 97-105 (1980). 


\title{
Supporting Information
}

\section{Unveiling the role of upper excited states in the photochemistry and laser performance of anti- $\mathrm{B}_{18} \mathrm{H}_{22}$.}

\author{
Luis Cerdán, ${ }^{1 *}$ Antonio Francés-Monerris, ${ }^{2,3}$ Daniel Roca-Sanjuán, ${ }^{4 *}$ Jonathan Bould, ${ }^{5}$ Jirri Dolanský, ${ }^{5}$ \\ Marcel Fuciman, ${ }^{6}$ and Michael G. S. Londesborough ${ }^{5 *}$
}

${ }^{1}$ Institute of Physical Chemistry "Rocasolano", Consejo Superior de Investigaciones Científicas (CSIC), C/ Serrano 119, 28006, Madrid, Spain

${ }^{2}$ Université de Lorraine and CNRS, LPCT, F-54000 Nancy, France

${ }^{3}$ Departament de Química Física, Universitat de València, Dr. Moliner 50, 46100 Burjassot, Spain

${ }^{4}$ Institut de Ciència Molecular, Universitat de València, P.O.Box 22085, 46010 Valencia - Spain

${ }^{5}$ Institute of Inorganic Chemistry of the Czech Academy of Sciences, 25068 Husinec-Řež, Czech Republic

${ }^{6}$ Institute of Physics, Faculty of Science, University of South Bohemia, Branišovská 1760, 37005 České Budějovice, Czech Republic

*lcerdanphd@gmail.com; *Daniel.Roca@uv.es; *michaell@iic.cas.cz

Section S1. Calculation of Excited State Absorption (ESA) cross section at the pump wavelength.

Section S2. Reconstruction of cross section spectra from oscillator strengths and transition energies.

Table S1. Vertical absorption and emission energies $\left(v_{i f}\right)$ (in $\mathrm{eV}$ and $\mathrm{nm}$ ) and associated oscillator strengths $\left(f_{i f}\right)$ between states $\mathrm{S}_{i}$ and $\mathrm{S}_{f}$ of anti- $\mathrm{B}_{18} \mathrm{H}_{22}$ at the $\mathrm{S}_{0}$ and $\mathrm{S}_{1}$ minimum geometry determined with two different methods (CASPT2 and MS- CASPT2).

Figure S1. ${ }^{11} \mathrm{~B}$ (above) and ${ }^{1} \mathrm{H}$ (below) NMR spectra of white precipitate from cuvette wall formed after continual $355 \mathrm{~nm}$ laser excitation.

Figure S2. Experimental set-ups for the intensity dependent transmission (a) and the long-irradiation (b) experiments

Figure S3. Photodegradation samples photographed before opening under visible white light

Figure S4. Direct comparison of samples 0mins (left) and 210mins (right).

Figure S5. Samples 0mins, 150mins and 210mins in cyclohexane solution in quartz NMR tubes under UV lamp

Figure S6. ${ }^{11} \mathrm{~B}-\left\{{ }^{1} \mathrm{H}\right\}$ NMR spectrum of reference sample $0 \operatorname{mins}(0 \mathrm{MJ})$ 
Figure S7. ${ }^{11} \mathrm{~B}-\left\{{ }^{1} \mathrm{H}\right\}$ NMR spectrum of sample $15 \operatorname{mins}(0.11 \mathrm{MJ})$

Figure S8. ${ }^{11} \mathrm{~B}-\left\{{ }^{1} \mathrm{H}\right\}$ NMR spectrum of sample $30 \operatorname{mins}(0.22 \mathrm{MJ})$

Figure S9. ${ }^{11} \mathrm{~B}-\left\{{ }^{1} \mathrm{H}\right\}$ NMR spectrum of sample $60 \operatorname{mins}(0.43 \mathrm{MJ})$

Figure S10. ${ }^{11} \mathrm{~B}-\left\{{ }^{1} \mathrm{H}\right\}$ NMR spectrum of sample $120 \mathrm{mins}(0.86 \mathrm{MJ})$

Figure S11. ${ }^{11} \mathrm{~B}-\left\{{ }^{1} \mathrm{H}\right\}$ NMR spectrum of sample $210 \operatorname{mins}(1.51 \mathrm{MJ})$

Figure S12. ${ }^{11} \mathrm{~B}-\left\{{ }^{1} \mathrm{H}\right\}$ NMR spectrum of sample $150 \mathrm{mins}(0.11 \mathrm{MJ})$

Figure S13. Full (top) and expanded (bottom) mass spectrum $210 \mathrm{~m}$ (1.15 MJ) sample of anti- $\mathrm{B}_{18} \mathrm{H}_{22}$.

Figure S14. CASSCF natural orbitals corresponding to the dominant excitations (configuration state functions) that characterise the $\mathrm{S}_{1}(\mathrm{a}), \mathrm{S}_{8}(\mathrm{~b}), \mathrm{S}_{11}(\mathrm{c})$, and $\mathrm{S}_{12}(\mathrm{~d})$ states.

Figure S15. ${ }^{11} \mathrm{~B}-\left\{{ }^{1} \mathrm{H}\right\}$ spectrum of anti- $\mathrm{B}_{18} \mathrm{H}_{22}$ sample irradiated for a period of 270 minutes

Figure S16. Mass spectrum of of anti- $\mathrm{B}_{18} \mathrm{H}_{22}$ sample irradiated for a period of 270 minutes

Figure S17. Reference (left-hand side) and 270 minute irradiated (right-hand side) samples of anti- $\mathrm{B}_{18} \mathrm{H}_{22}$ in $c$-hexane under UV lamp irradiation. 
Section S1. Calculation of Excited State Absorption (ESA) cross section at the pump wavelength.

We first assume that a Gaussian pulse with a pulse full width half maximum $\tau_{p}(7 \mathrm{~ns})$ is travelling downwards (Z-axis) and impinges on the upper surface of the solution at $\mathrm{Z}=0$ (Equation $\mathrm{S} 1$ ). The photon flux (\#photons $\mathrm{s}^{-1} \mathrm{~cm}^{-2}$ ) in this point will follow the expression:

$$
I_{p}(z=0, t)=I_{p}^{0} e^{-\frac{\left(t-t_{0}\right)^{2}}{\left(\tau_{p} / 2\right)^{2}} \log 2}
$$

As it propagates within an absorptive medium of length $\mathrm{L}$, it will be absorbed by the populations of the ground state $\left(N_{0}\right)$ and the first $\left(N_{I}\right)$ and upper excited states $\left(N_{N}\right)$. Thus, the pump photon flux follows the spatio-temporal equation:

$$
\begin{aligned}
\frac{d I_{p}(z, t)}{d z}= & \frac{n}{c} \frac{\partial I_{p}(z, t)}{\partial t}+\frac{\partial I_{p}(z, t)}{\partial z}= \\
& -\sigma_{0 p} N_{0}(z, t) I_{p}(z, t)-\sigma_{1 p} N_{1}(z, t) I_{p}(z, t)-\sigma_{N p} N_{N}(z, t) I_{p}(z, t)
\end{aligned}
$$

where $n$ is the medium refractive index (1.43), $c$ the speed of light in vacuum, $\sigma_{0 p}, \sigma_{l p}$ and $\sigma_{N p}$ are the ground $\left(\mathrm{S}_{0}\right)$ and $\mathrm{S}_{1}, \mathrm{~S}_{\mathrm{N}}$ excited-state absorption cross-sections at the pump wavelength, and $N_{0}, N_{l}$ and $N_{N}$ are the volume density of molecules in the $\mathrm{S}_{0}, \mathrm{~S}_{1}$, and $\mathrm{S}_{\mathrm{N}}$ states. The populations $N_{i}$ (with $i=0,1, N$ ) evolve in time and space as:

$$
\begin{aligned}
& \frac{d N_{N}(z, t)}{d t}=\sigma_{1 p} N_{1}(z, t) I_{p}(z, t)-\frac{N_{N}(z, t)}{\tau_{N}}-\sigma_{N p} N_{N}(z, t) I_{p}(z, t) ; \\
& \frac{d N_{1}(z, t)}{d t}=\sigma_{0 p} N_{0}(z, t) I_{p}(z, t)-\frac{N_{1}(z, t)}{\tau}-\sigma_{1 p} N_{1}(z, t) I_{p}(z, t)+\frac{N_{N}(z, t)}{\tau_{N}} ; \\
& N_{d}=N_{0}(z, t)+N_{1}(z, t)+N_{N}(z, t)
\end{aligned}
$$

where $\tau_{N}$ and $\tau$ are the life-times of the upper and first excited states, respectively. $N_{d}$ is the volume density of active molecules in the solution. In the present approach, we will assume, as a reasonable approximation, that the pump photon flux is not absorbed by upper excited states $\mathrm{S}_{\mathrm{N}}$, and therefore $\sigma_{N p}=0$. This implies that, effectively, the whole of the excited state absorption takes place in the first excited state $\mathrm{S}_{1}$. The population and intensity propagation equations (S2) and (S3) then simplifies to:

$$
\begin{aligned}
& \frac{d I_{p}(z, t)}{d z}=\frac{n}{c} \frac{\partial I_{p}(z, t)}{\partial t}+\frac{\partial I_{p}(z, t)}{\partial z}=-\sigma_{0 p} N_{0}(z, t) I_{p}(z, t)-\sigma_{1 p} N_{1}(z, t) I_{p}(z, t) \\
& \frac{d N_{N}(z, t)}{d t}=\sigma_{1 p} N_{1}(z, t) I_{p}(z, t)-\frac{N_{N}(z, t)}{\tau_{N}} \\
& \frac{d N_{1}(z, t)}{d t}=\sigma_{0 p} N_{0}(z, t) I_{p}(z, t)-\frac{N_{1}(z, t)}{\tau}-\sigma_{1 p} N_{1}(z, t) I_{p}(z, t)+\frac{N_{N}(z, t)}{\tau_{N}} \\
& N_{d}=N_{0}(z, t)+N_{1}(z, t)+N_{N}(z, t)
\end{aligned}
$$


Numerically solving the system of coupled differential equations (S4) with boundary conditions (S1), allows obtaining the pump photon flux exiting the medium $I_{p}(z=L, t)$. Thus, the numerically computed value of transmission is given by:

$$
T=100 \% \frac{\int I_{p}(z=L, t) d t}{\int I_{p}(z=0, t) d t}
$$

To compute the proportion of pump photons that are absorbed due to Ground State Absorption (GSA) or Excited State Absorption (ESA), the following propagation equations are solved in parallel with the system of eqs. (S4):

$$
\begin{aligned}
& \frac{d I_{p, G S A}(z, t)}{d z}=\frac{n}{c} \frac{\partial I_{p, G S A}(z, t)}{\partial t}+\frac{\partial I_{p, G S A}(z, t)}{\partial z}=-\sigma_{0 p} N_{0}(z, t) I_{p, G S A}(z, t) \\
& \frac{d I_{p, E S A}(z, t)}{d z}=\frac{n}{c} \frac{\partial I_{p, E S A}(z, t)}{\partial t}+\frac{\partial I_{p, E S A}(z, t)}{\partial z}=-\sigma_{1 p} N_{1}(z, t) I_{p, E S A}(z, t)
\end{aligned}
$$

using the values of $N_{0}$ and $N_{l}$ updated while solving the system of eqs. (S4). The proportion of photons absorbed due to GSA or ESA is then given by:

$$
\begin{aligned}
& \alpha_{G S A}=1-\frac{\int I_{p, G S A}(z=L, t) d t}{\int I_{p, G S A}(z=0, t) d t} \\
& \alpha_{E S A}=1-\frac{\int I_{p, E S A}(z=L, t) d t}{\int I_{p, E S A}(z=0, t) d t}
\end{aligned}
$$

It is easy to see that the total amount of absorbed pump photons $(\alpha=1-T / 100)$ must be the sum of the amount of photons absorbed by GSA and ESA, i.e., $\alpha=\alpha_{G S A}+\alpha_{E S A}$. Thus, the percentage of absorbed photons that goes into ESA, $\rho_{E S A}$, is calculated as:

$$
\rho_{E S A}=\frac{\alpha_{E S A}}{\alpha_{G S A}+\alpha_{E S A}}
$$


Section S2. Reconstruction of cross section spectra from oscillator strengths and transition energies.

Computing the transitions band shape in a formal way would involve determining the vibronic couplings between all the electronic states. Even though for the lowest-lying states $\left(S_{0}\right.$ and $\left.S_{1}\right)$ this would be reasonable to do, for upper excited states, with their strong multiconfigurational character, large anharmonicity and many near-degeneracies, accurate and reliable computations of the vibronic couplings within the CASSCF/(MS-)CASPT2 approach become prohibitive. Accordingly, in the present work, we opted for a simpler, but standard, approach ${ }^{\mathrm{S} 1, \mathrm{~S} 2}$ based on assigning each computed transition with a Gaussian function the proportionality factor of which is determined by the oscillator strength and transition energy. In an ideal case, each transition would have to be assigned its own line-width, but it is qualitatively sufficient to assign the same line-width to all transitions. This line-width $\Delta v$ is usually chosen to replicate the experimentally available absorption bands line-widths. To get an improved prediction of the bands line-shapes, nuclear sampling of the ground-state Franck-Condon minimum could be done with a semi-classical Wigner distribution based on the frequencies and subsequently convolving each point of the distribution with Gaussian functions. ${ }^{\mathrm{S} 3}$ Nevertheless, since experimental spectroscopic data is available in our study, the single electronic transition at the equilibrium structures of $S_{0}$ and $S_{1}$, and correlation to the measured spectra, is sufficient for the objectives of this work, avoiding very high computational demands that would be required in the relatively large-size of the octadecaborane molecule that we study herein.

The proportionality factor is computed as follows. The oscillator strength for an electronic transition between an initial state $i$ and a final state $f\left(f_{i f}\right)$ and the molar extinction coefficient $\varepsilon_{i j}$ spectrum (in $\left.\mathrm{M}^{-1} \mathrm{~cm}^{-1}\right)$ of a given transition centred at an energy $v_{i f}$ are related through the common expression: ${ }^{\mathrm{S4}}$

$$
f_{i f}=8065.5 \cdot 4.32 \times 10^{-9} F\left(n, v_{i f}\right) \int \varepsilon_{i j} d v
$$

The leading 8065.5 factor has been included to take into account that the energy $v$ is expressed in $\mathrm{eV}$ instead of $\mathrm{cm}^{-1}$, as is usually done. $F\left(n, v_{i f}\right)$ is the local field factor that takes the solvent effects into account. In the Onsager real cavity approximation ${ }^{\mathrm{S} 5} F\left(n, v_{i f}\right)=\left(2 n\left(v_{i f}\right)^{2}+1\right)^{2} /\left(9 n\left(v_{i f}\right)^{3}\right)$, with $n\left(v_{i f}\right)$ being the solvent refractive index at transition energy $v_{i f}$. The molar extinction coefficient, $\varepsilon_{i f}$, is defined as a Gaussian function:

$$
\varepsilon_{i j}(v)=\frac{\varepsilon_{\max }}{\sqrt{2 \pi} \Delta v / 2.3548} e^{-\frac{\left(v-v_{i j}\right)^{2}}{2(\Delta v / 2.3548)^{2}}}
$$

so that $\int \varepsilon_{i j} \mathrm{~d} v=\varepsilon_{\max }$. Substituting (S10) into (S9), and solving for $\varepsilon_{\max }$, leads to:

$$
\varepsilon_{\max }=\frac{f_{i f}}{8065.5 \cdot 4.32 \times 10^{-9} F\left(n, v_{i f}\right)}
$$

On the other hand, the absorption cross section, $\sigma_{i f}(v)$, (in $\left.\mathrm{cm}^{2}\right)$ and the $\varepsilon_{i f}$, (in $\left.\mathrm{M}^{-1} \mathrm{~cm}^{-1}\right)$ are related as:

$$
\sigma_{i j}(v)=\ln (10) \cdot 1000 \frac{\varepsilon_{i j}(v)}{N_{A}}
$$

where $\mathrm{N}_{\mathrm{A}}$ is Avogrado's number. Substituting (S11) into (S10) and the resulting expression into (S12), leads 
to the absorption cross section of each transition:

$$
\sigma_{i j}(v)=\frac{\ln (10) \cdot 1000}{N_{A} \sqrt{2 \pi} \Delta v / .3548} \frac{f_{i j}}{8065.4 \cdot 4.32 \times 10^{-9} F\left(n, v_{i j}\right)} e^{-\frac{\left(v-v_{i j}\right)^{2}}{2(\Delta v / 2.3548)^{2}}}
$$

The absorption spectrum of the initial state $i$ is thus obtained summing up the individual contributions of each transition:

$$
\sigma_{i}(v)=\sum_{j} \sigma_{i j}(v)=\sum_{j} 1.03 \times 10^{-16} \frac{f_{i j}}{F\left(n, v_{i j}\right) \Delta v} e^{-\frac{\left(v-v_{i j}\right)^{2}}{2(\Delta v / 2.3548)^{2}}}
$$

Equation (S14) thus constructs the cross-section spectrum from oscillator strengths and transition energies. The indexes $i$ and $f$ run as follows for each type of spectrum determined in this work: for GSA, $i=0$ and $f=$ $1 \ldots N$, and for ESA, $i=1$ and $f=2 \ldots N_{t o t}$, where $N_{\text {tot }}$ is the total number of electronic states considered.

\section{References}

S1. R. Rüger, E. van Lenthe, Y. Lu, J. Frenzel, T. Heine and L. Visscher, "Efficient Calculation of Electronic Absorption Spectra by Means of Intensity-Selected Time-Dependent Density Functional Tight Binding," J. Chem. Theory Comput. 11, 157-167 (2015).

S2. E. F Oliveira, J. Shi, F. C. Lavarda, L. Lüer, B. Milián-Medina, and J. Gierschner, "Excited state absorption spectra of dissolved and aggregated distyrylbenzene: A TD-DFT state and vibronic analysis" J. Chem. Phys. 147, 034903 (2017).

S3. E. Wigner, Phys. Rev. 40 (1932) 749.

S4. J. R. Lakowicz, Principles of Fluorescence Spectroscopy $3^{\text {rd }}$ Ed. (Springer, 2006).

S5. K. Dolgaleva and R. W. Boyd, "Local-field effects in nanostructured photonic materials," Adv. Opt. Photon. 4, 1-77 (2012). 
Table S1. Vertical absorption and emission energies $\left(v_{i f}\right)$ (in $\mathrm{eV}$ and $\mathrm{nm}$ ) and associated oscillator strengths $\left(f_{i f}\right)$ between states $\mathrm{S}_{i}$ and $\mathrm{S}_{f}$ of anti- $\mathrm{B}_{18} \mathrm{H}_{22}$ at the $\mathrm{S}_{0}$ and $\mathrm{S}_{1}$ minimum geometry determined with two different methods (CASPT2 and MS- CASPT2).

\begin{tabular}{|c|c|c|c|c|c|c|}
\hline \multirow[b]{2}{*}{ Transition } & \multicolumn{3}{|c|}{ CASPT2 } & \multicolumn{3}{|c|}{ MS-CASPT2 } \\
\hline & $v_{i f}(\mathrm{eV})$ & $v_{i f}(\mathbf{n m})$ & $f_{i f}$ & $v_{i f}(\mathrm{eV})$ & $v_{i f}(\mathbf{n m})$ & $f_{i f}$ \\
\hline \multicolumn{7}{|c|}{ absorption at the $\mathrm{S}_{0}$-minimum geometry (GSA) } \\
\hline $\mathbf{S}_{\mathbf{0}} \rightarrow \mathbf{S}_{\mathbf{1}}$ & 3.93 & 315 & 0.2917 & 3.93 & 315 & 0.3136 \\
\hline $\mathbf{S}_{\mathbf{0}} \rightarrow \mathbf{S}_{2}$ & 5.00 & 248 & 0.1709 & 4.99 & 248 & 0.1676 \\
\hline $\mathbf{S}_{\mathbf{0}} \rightarrow \mathbf{S}_{3}$ & 5.03 & 246 & 0.0000 & 5.02 & 247 & 0.0000 \\
\hline $\mathbf{S}_{\mathbf{0}} \rightarrow \mathbf{S}_{4}$ & 5.35 & 232 & 0.0000 & 5.33 & 233 & 0.0000 \\
\hline $\mathbf{S}_{\mathbf{0}} \rightarrow \mathbf{S}_{\mathbf{5}}$ & 5.43 & 228 & 0.0000 & 5.42 & 229 & 0.0000 \\
\hline $\mathbf{S}_{0} \rightarrow \mathbf{S}_{6}$ & 5.52 & 225 & 0.0390 & 5.54 & 224 & 0.0283 \\
\hline $\mathbf{S}_{\mathbf{0}} \rightarrow \mathbf{S}_{7}$ & 5.76 & 215 & 0.0000 & 5.80 & 214 & 0.0000 \\
\hline \multicolumn{7}{|c|}{ absorption at the $\mathrm{S}_{1}$-minimum geometry (ESA) } \\
\hline $\mathbf{S}_{1} \rightarrow \mathbf{S}_{2}$ & 1.31 & 944 & 0.0421 & 1.25 & 992 & 0.0523 \\
\hline $\mathbf{S}_{1} \rightarrow \mathbf{S}_{3}$ & 1.54 & 808 & 0.0000 & 1.51 & 824 & 0.0000 \\
\hline $\mathbf{S}_{\mathbf{1}} \rightarrow \mathbf{S}_{\mathbf{4}}$ & 1.63 & 763 & 0.0000 & 1.64 & 757 & 0.1035 \\
\hline $\mathbf{S}_{\mathbf{1}} \rightarrow \mathbf{S}_{\mathbf{5}}$ & 1.71 & 725 & 0.0766 & 1.68 & 740 & 0.0000 \\
\hline $\mathbf{S}_{1} \rightarrow \mathbf{S}_{6}$ & 1.77 & 700 & 0.0191 & 1.89 & 655 & 0.0665 \\
\hline $\mathbf{S}_{1} \rightarrow \mathbf{S}_{7}$ & 2.17 & 570 & 0.0271 & 2.22 & 560 & 0.0030 \\
\hline $\mathbf{S}_{1} \rightarrow \mathbf{S}_{8}$ & 2.60 & 476 & 0.3509 & 2.67 & 464 & 0.3658 \\
\hline $\mathbf{S}_{1} \rightarrow \mathbf{S}_{9}$ & 3.02 & 410 & 0.0000 & 3.03 & 409 & 0.0000 \\
\hline $\mathbf{S}_{1} \rightarrow \mathbf{S}_{10}$ & 3.25 & 382 & 0.0000 & 3.29 & 376 & 0.0000 \\
\hline$S_{1} \rightarrow S_{11}$ & 3.33 & 373 & 0.0902 & 3.39 & 366 & 0.0145 \\
\hline$S_{1} \rightarrow S_{12}$ & 3.61 & 344 & 0.0304 & 3.66 & 339 & 0.0048 \\
\hline$S_{1} \rightarrow S_{13}$ & 3.96 & 313 & 0.0000 & 3.95 & 314 & 0.0000 \\
\hline$S_{1} \rightarrow S_{14}$ & 4.10 & 303 & 0.0000 & 4.11 & 302 & 0.0000 \\
\hline$S_{1} \rightarrow S_{15}$ & 4.20 & 295 & 0.1090 & 4.17 & 297 & 0.0991 \\
\hline $\mathbf{S}_{1} \rightarrow \mathbf{S}_{16}$ & 4.22 & 294 & 0.0000 & 4.40 & 282 & 0.0000 \\
\hline $\mathbf{S}_{1} \rightarrow \mathbf{S}_{17}$ & 4.41 & 281 & 0.0596 & 4.52 & 275 & 0.0279 \\
\hline $\mathbf{S}_{1} \rightarrow \mathbf{S}_{18}$ & 4.53 & 274 & 0.0530 & 4.68 & 265 & 0.0000 \\
\hline $\mathbf{S}_{1} \rightarrow \mathbf{S}_{19}$ & 4.54 & 273 & 0.0130 & 4.71 & 263 & 0.0018 \\
\hline \multicolumn{7}{|c|}{ emission at the $\mathbf{S}_{\mathbf{1}}$ minimum geometry (SE) } \\
\hline $\mathbf{S}_{1} \rightarrow \mathbf{S}_{\mathbf{0}}$ & 3.00 & 413 & 0.3279 & 2.97 & 417 & 0.4356 \\
\hline
\end{tabular}


Figure S1. ${ }^{11} \mathrm{~B}$ (above) and ${ }^{1} \mathrm{H}$ (below) NMR spectra of white precipitate from cuvette wall formed after continual $355 \mathrm{~nm}$ laser excitation.
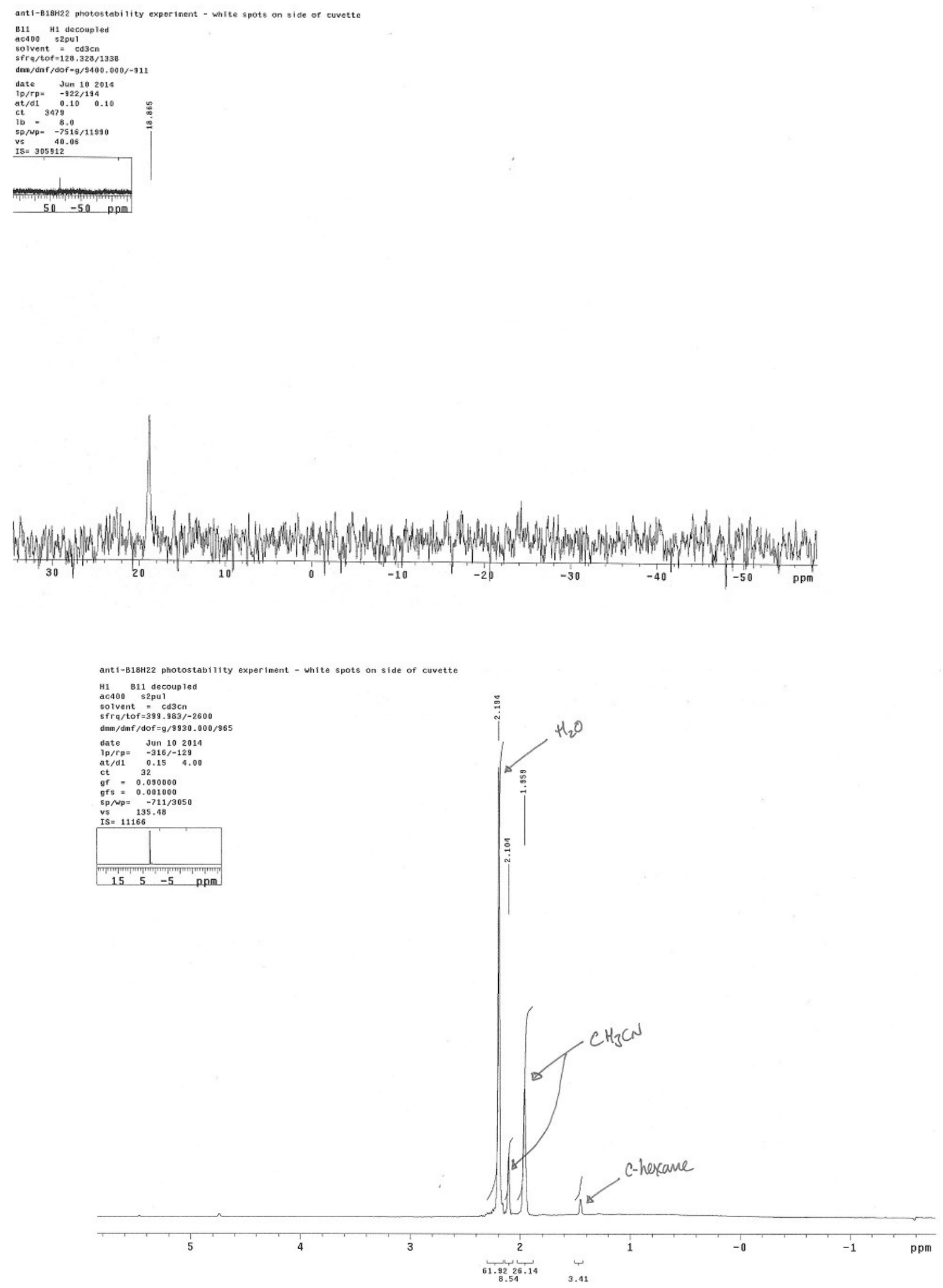
Figure S2: Experimental set-ups for the intensity dependent transmission (a) and the long-irradiation (b) experiments.

a)

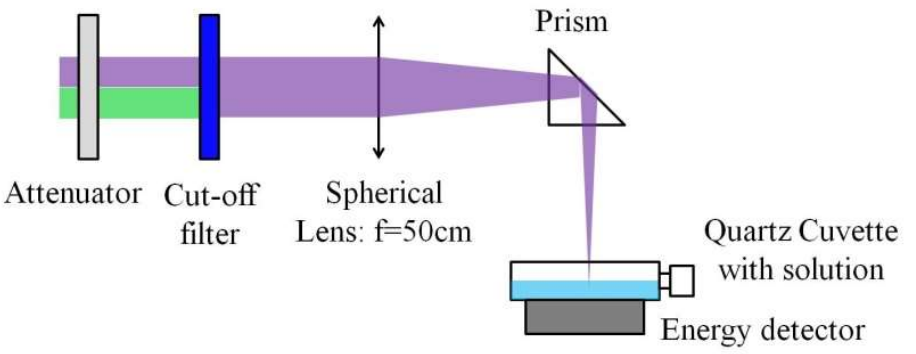

b)

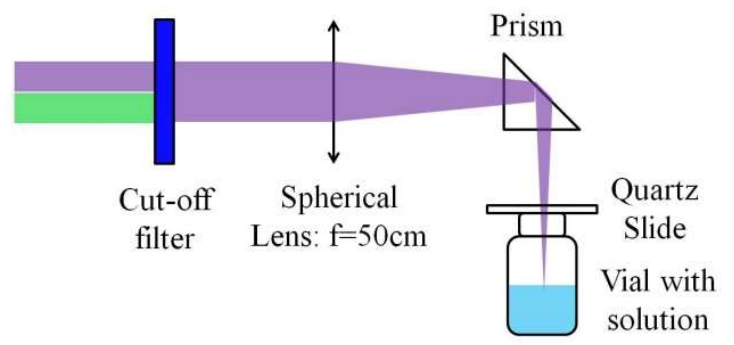

Figure S3. Photodegradation samples photographed before opening under visible white light

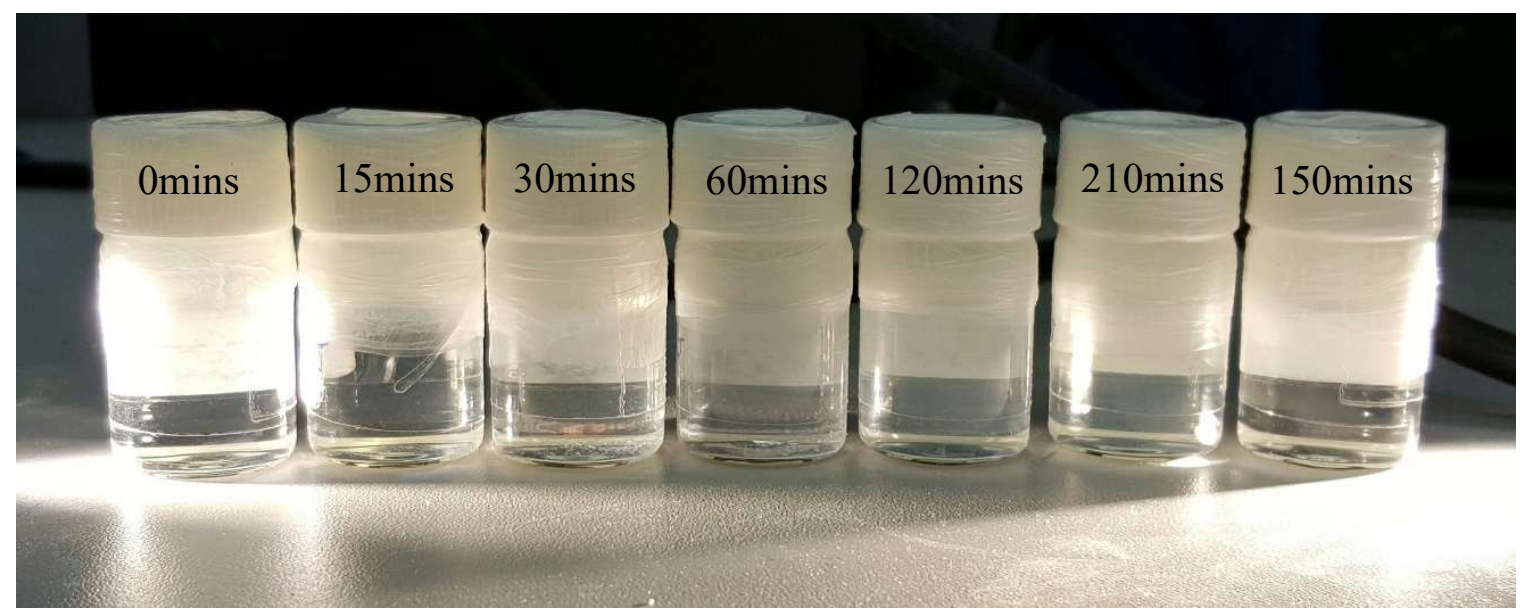


Figure S4. Direct comparison of samples 210mins (left) and 0mins (right). Note the slight yellowish hue and traces of white precipitate at the bottom of the left-hand vial.

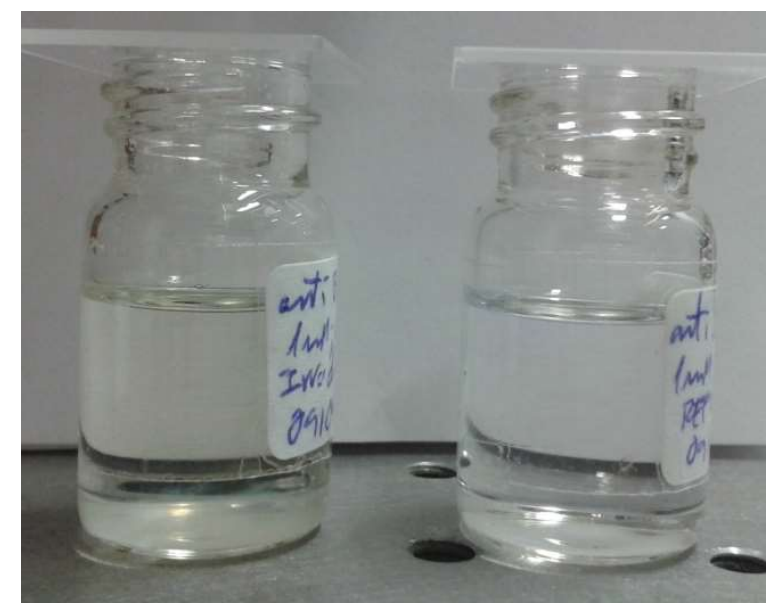

Figure S5. Samples 0mins, 150mins and 210mins in cyclohexane solution in quartz NMR tubes under UV lamp

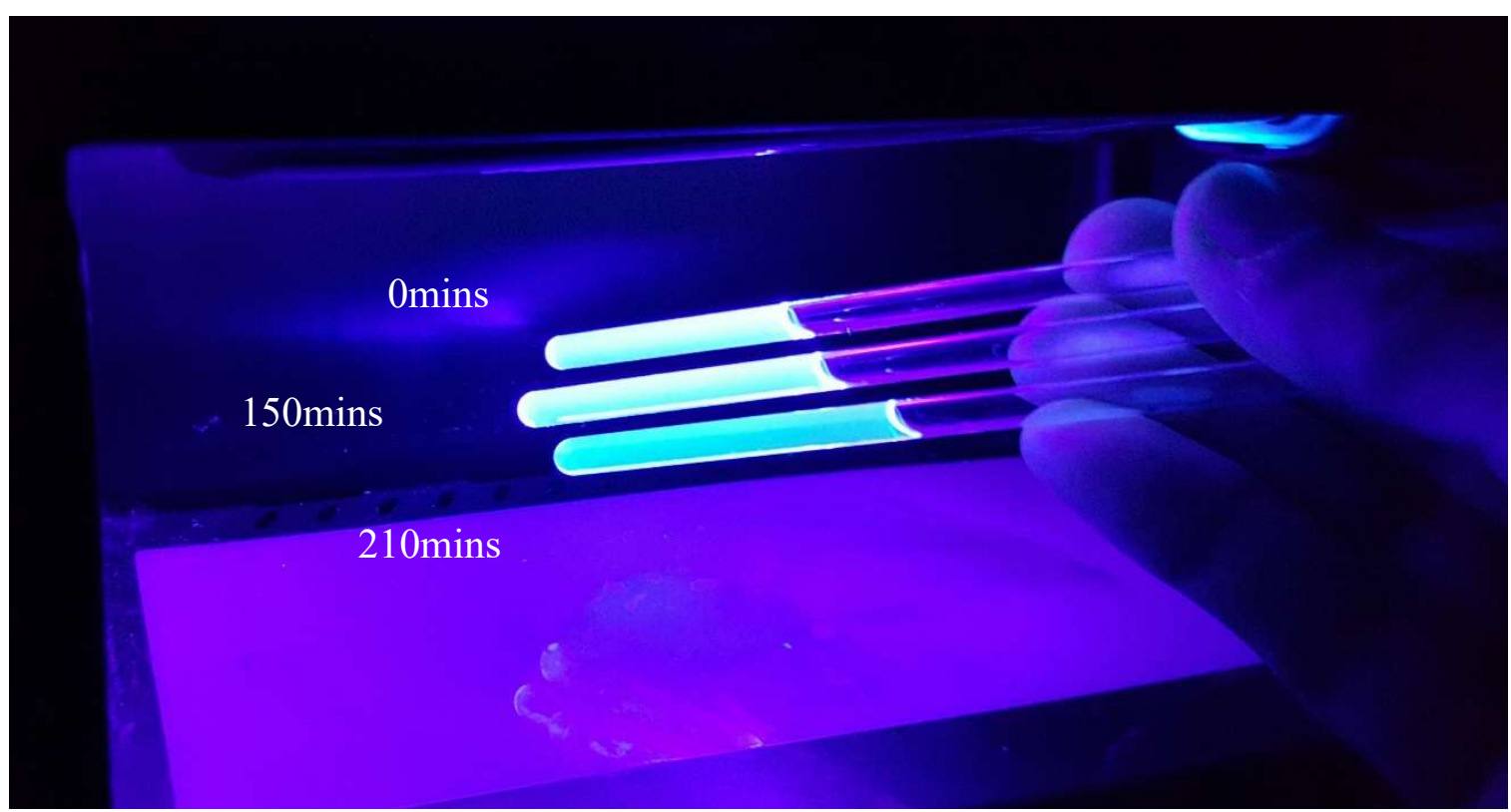


Figure S6. ${ }^{11} \mathrm{~B}-\left\{{ }^{1} \mathrm{H}\right\}$ NMR spectrum of reference sample $0 \operatorname{mins}(0 \mathrm{MJ})$

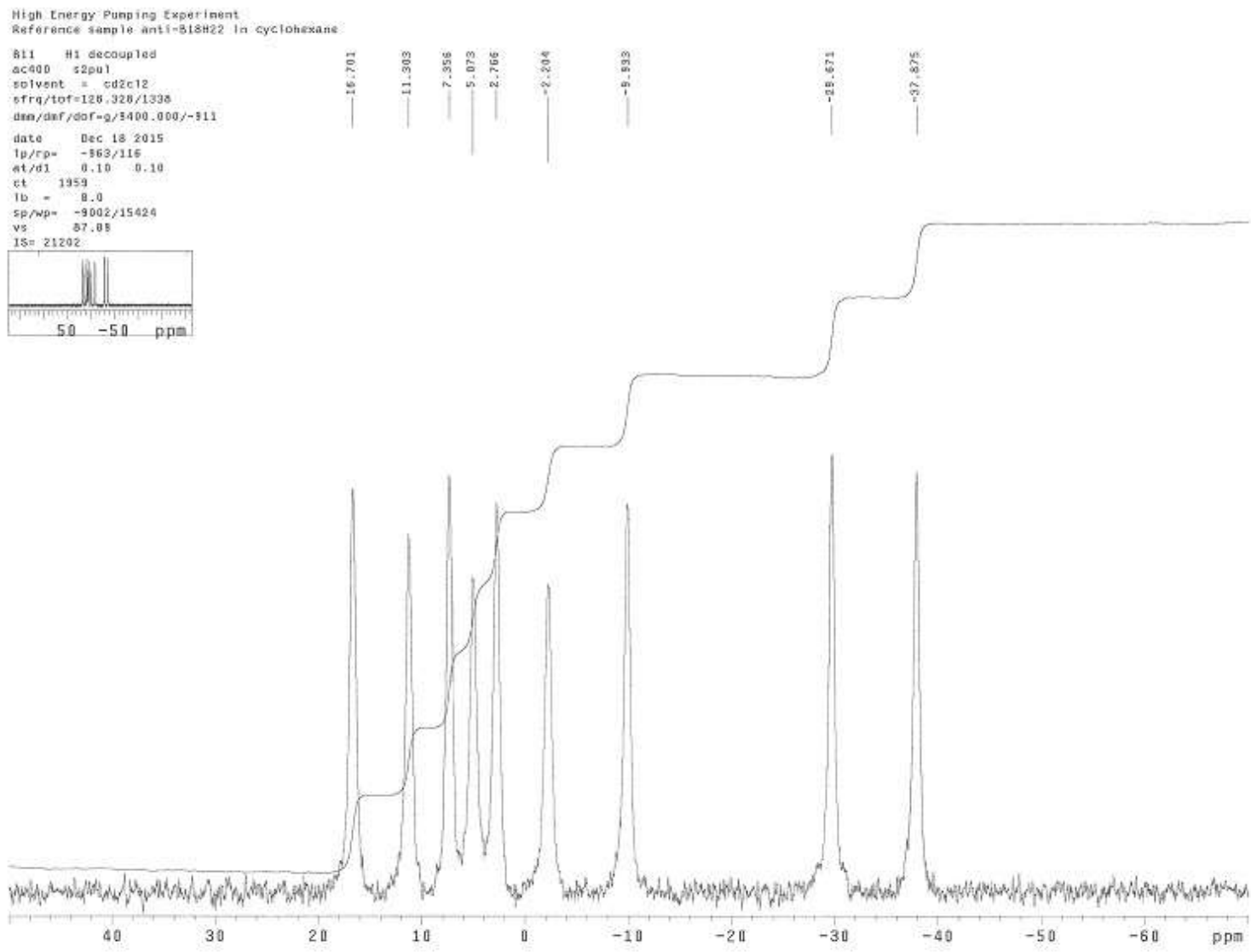

Figure S7. ${ }^{11} \mathrm{~B}-\left\{{ }^{1} \mathrm{H}\right\}$ NMR spectrum of sample $15 \operatorname{mins}(0.11 \mathrm{MJ})$

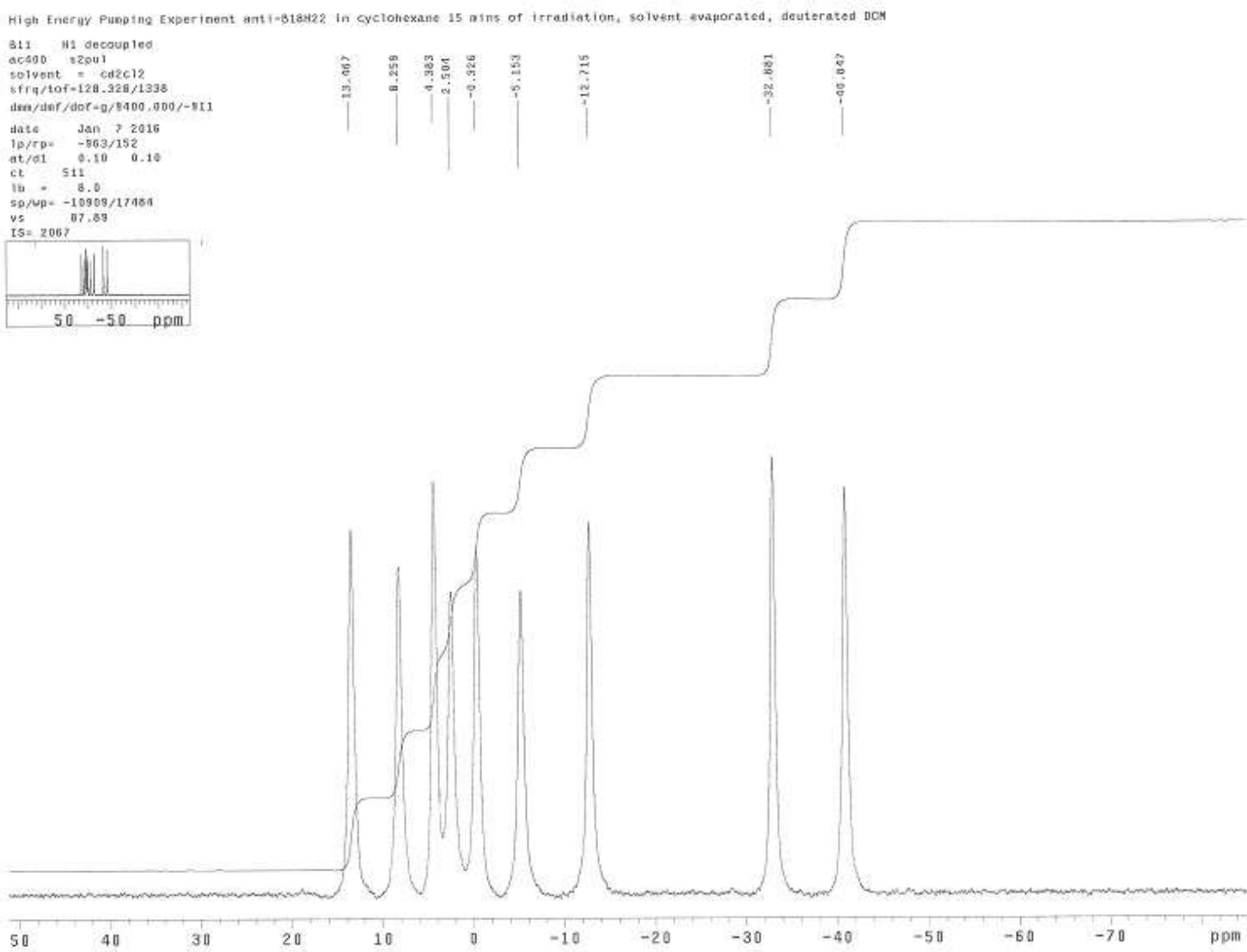


Figure S8. ${ }^{11} \mathrm{~B}-\left\{{ }^{1} \mathrm{H}\right\}$ NMR spectrum of sample $30 \operatorname{mins}(0.22 \mathrm{MJ})$

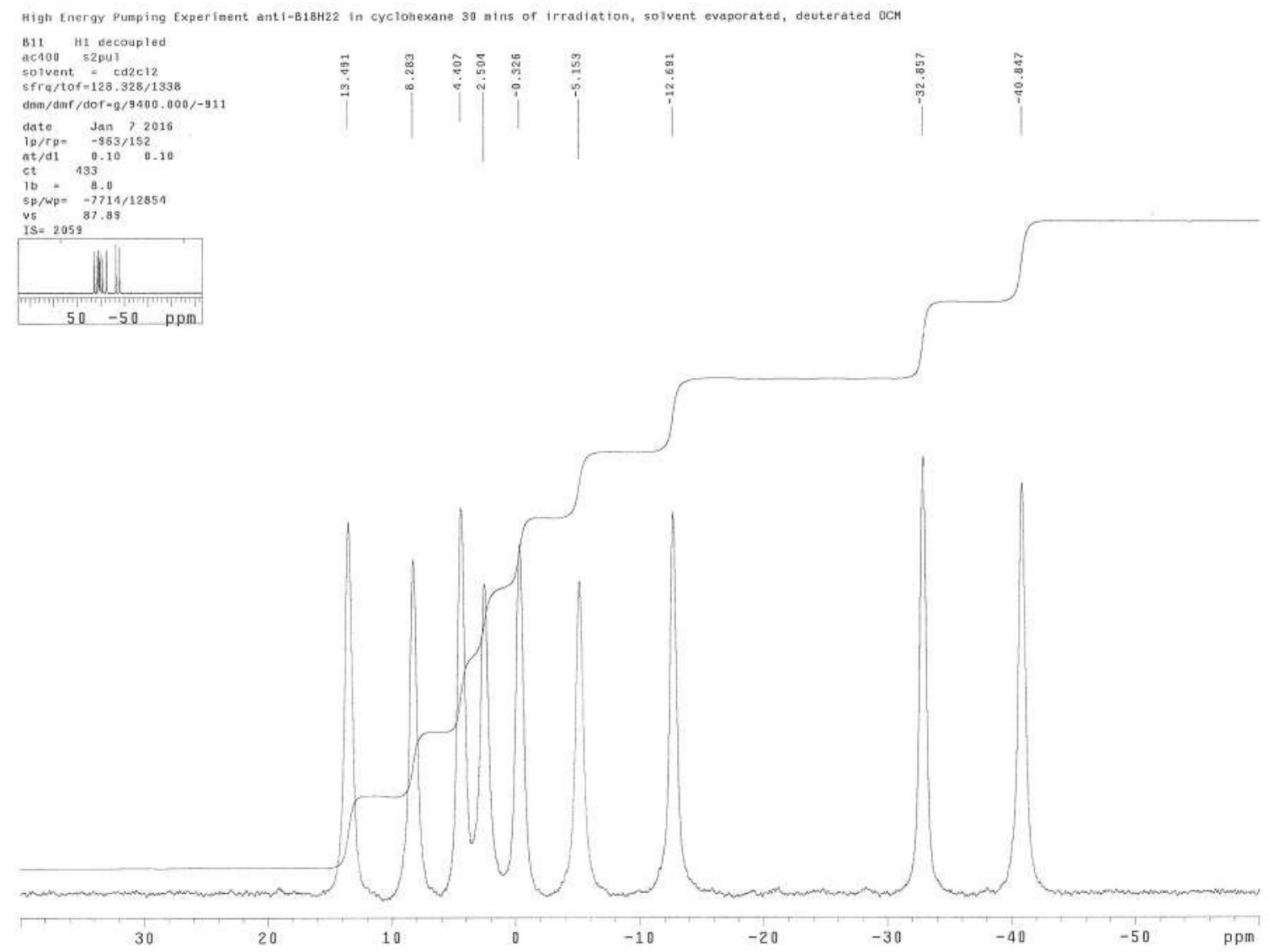

Figure S9. ${ }^{11} \mathrm{~B}-\left\{{ }^{1} \mathrm{H}\right\}$ NMR spectrum of sample $60 \operatorname{mins}(0.43 \mathrm{MJ})$

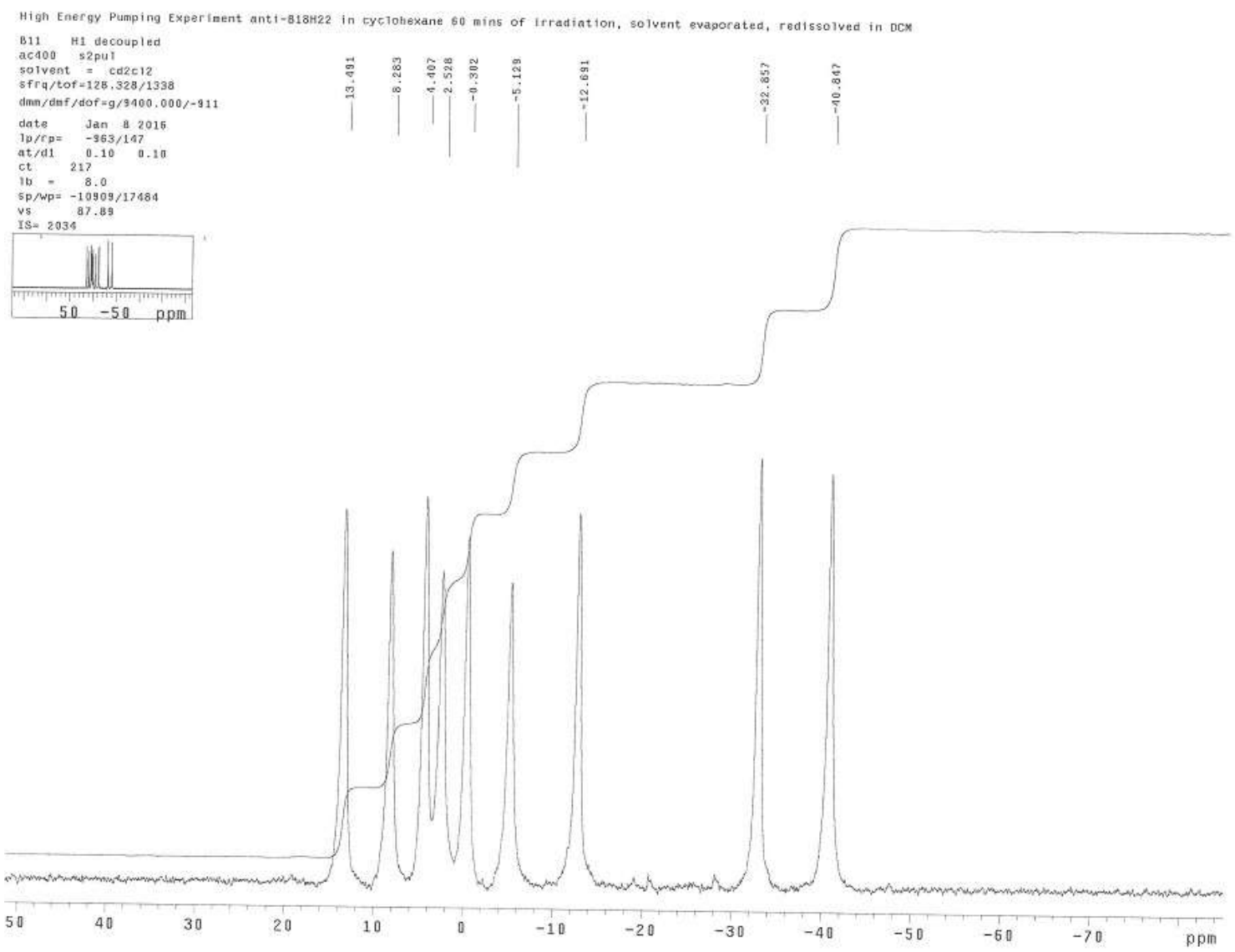


Figure S10. ${ }^{11} \mathrm{~B}-\left\{{ }^{1} \mathrm{H}\right\}$ NMR spectrum of sample $120 \operatorname{mins}(0.86 \mathrm{MJ})$

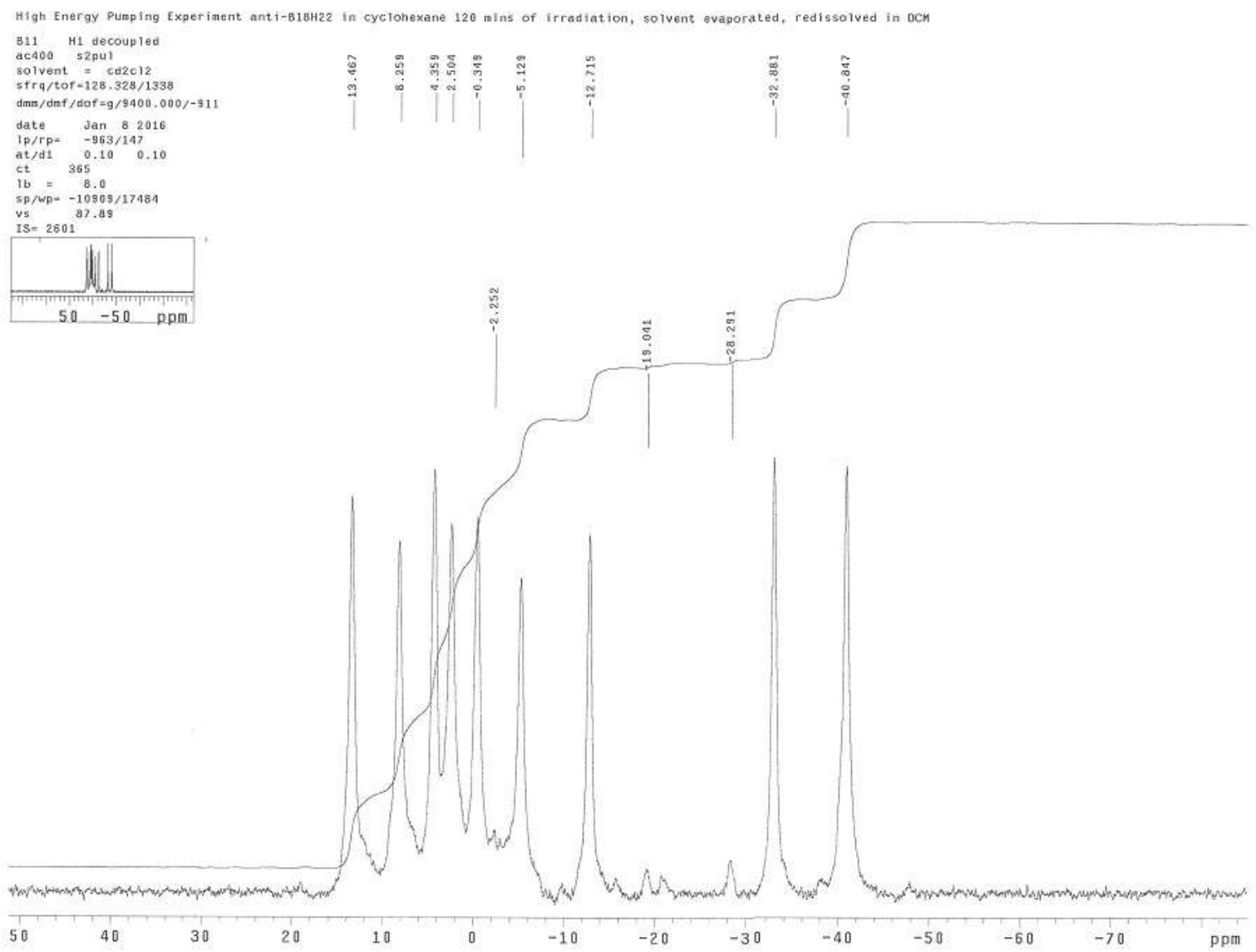

Figure S11. ${ }^{11} \mathrm{~B}-\left\{{ }^{1} \mathrm{H}\right\}$ NMR spectrum of sample 210mins(1.51 MJ)

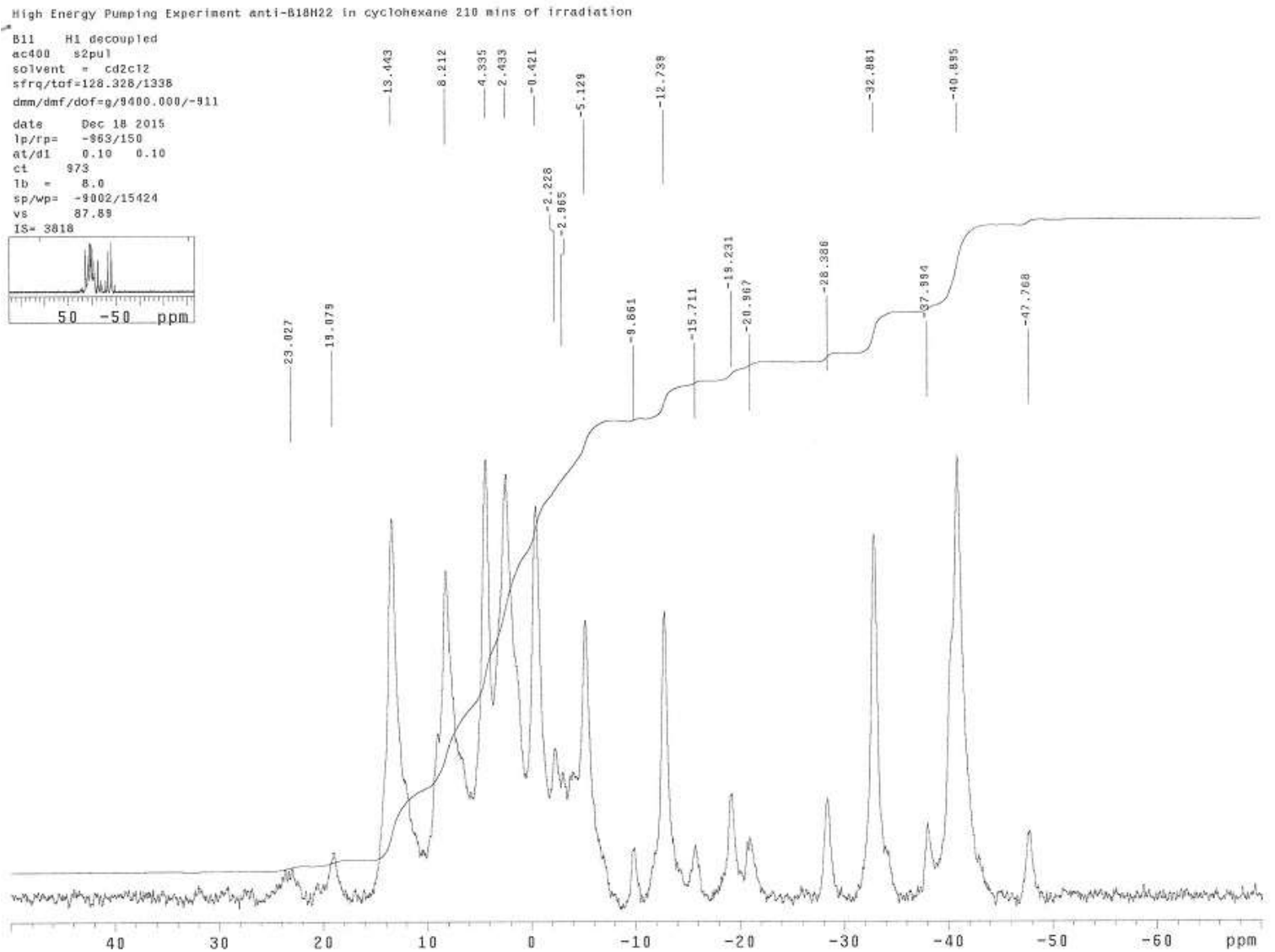


Figure S12. ${ }^{11} \mathrm{~B}-\left\{{ }^{1} \mathrm{H}\right\}$ NMR spectrum of sample $150 \mathrm{mins}(0.11 \mathrm{MJ})$

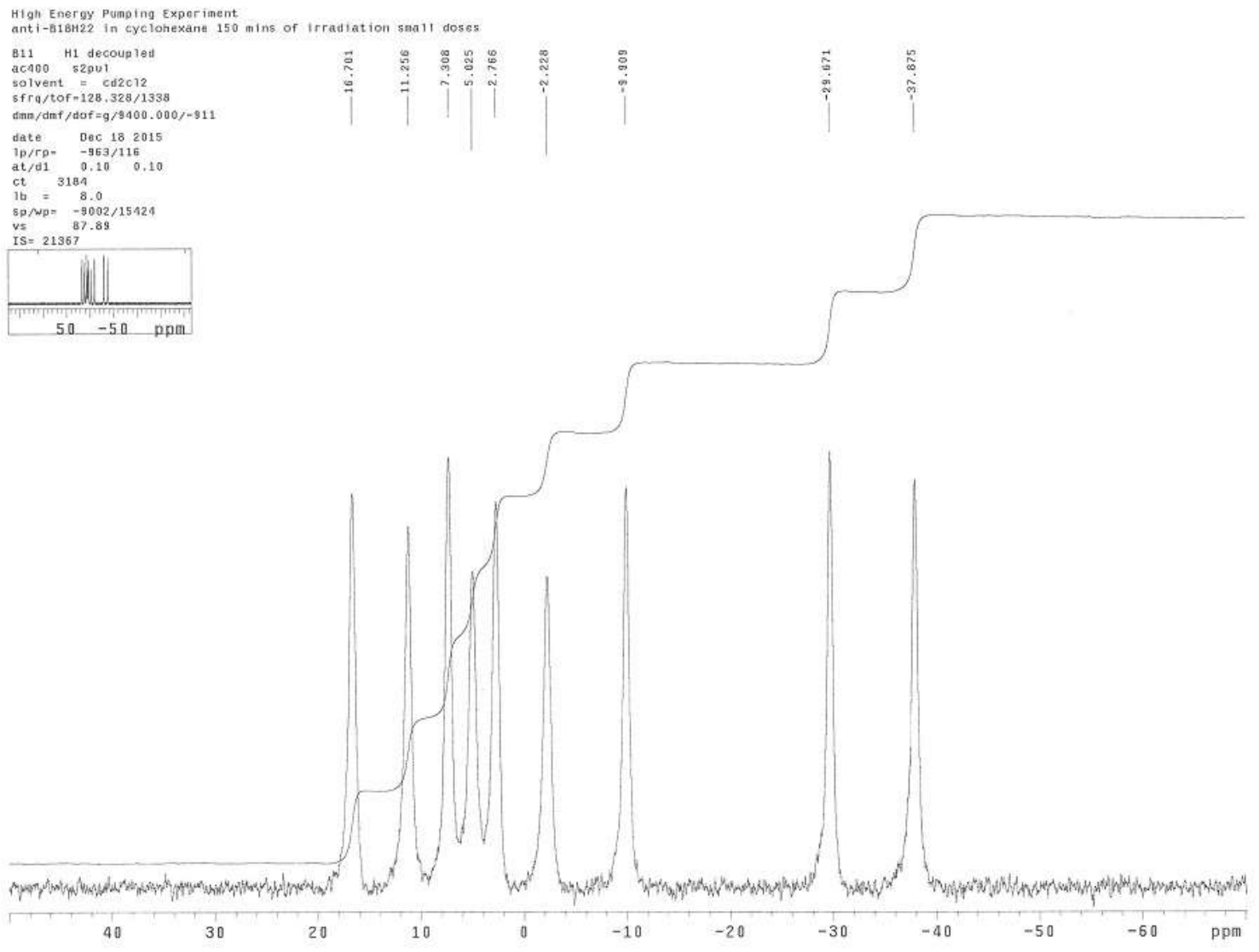


Figure S13. Full (top) and expanded (bottom) mass spectrum $210 \mathrm{~m}$ (1.15 MJ) sample of anti- $\mathrm{B}_{18} \mathrm{H}_{22}$.
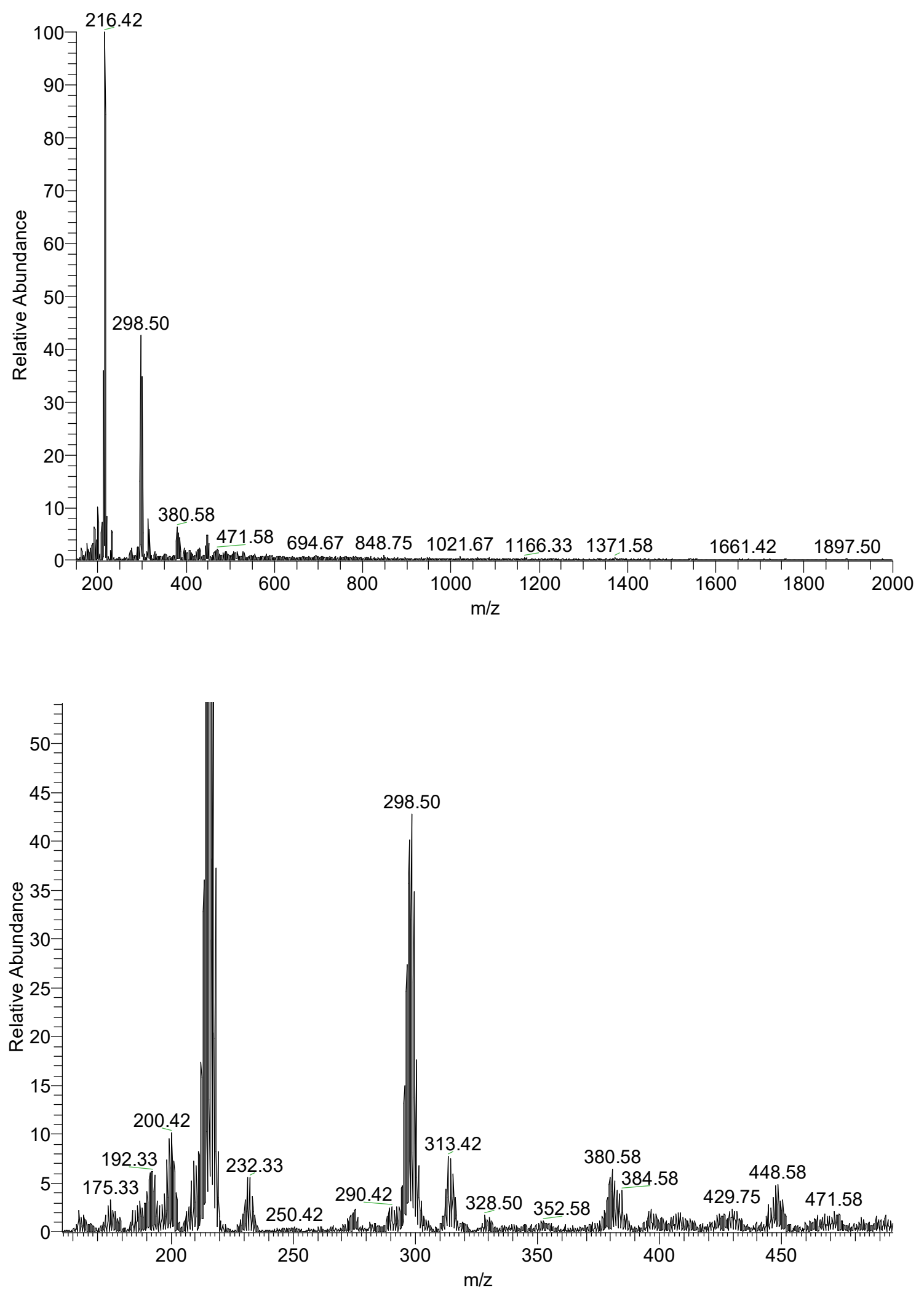
Figure S14. CASSCF natural orbitals corresponding to the dominant excitations (configuration state functions) that characterise the $S_{1}(a), S_{8}(b), S_{11}(c)$, and $S_{12}(d)$ states. Dotted yellow lines indicate the bonds with antibonding character, and $\mathbf{B}$ refers to the conjuncto boron atoms that are shared by the molecule's two subclusters.

a)
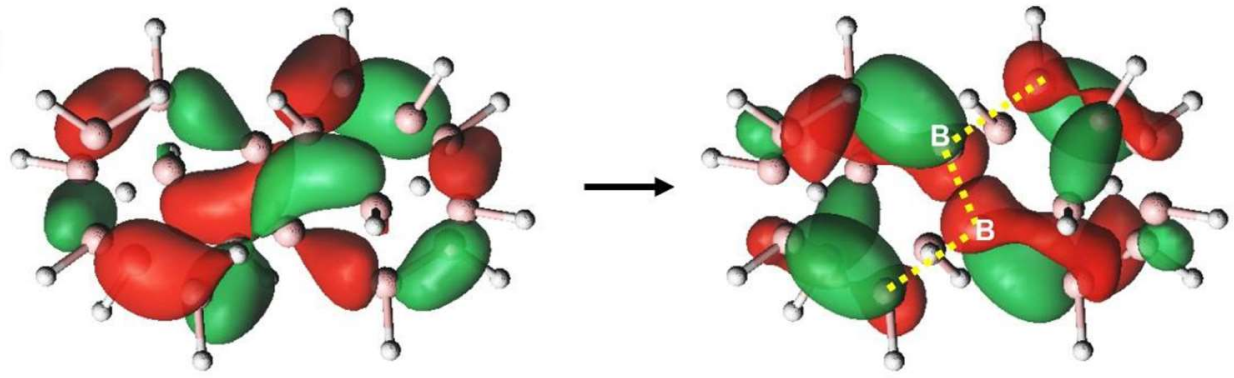

b)
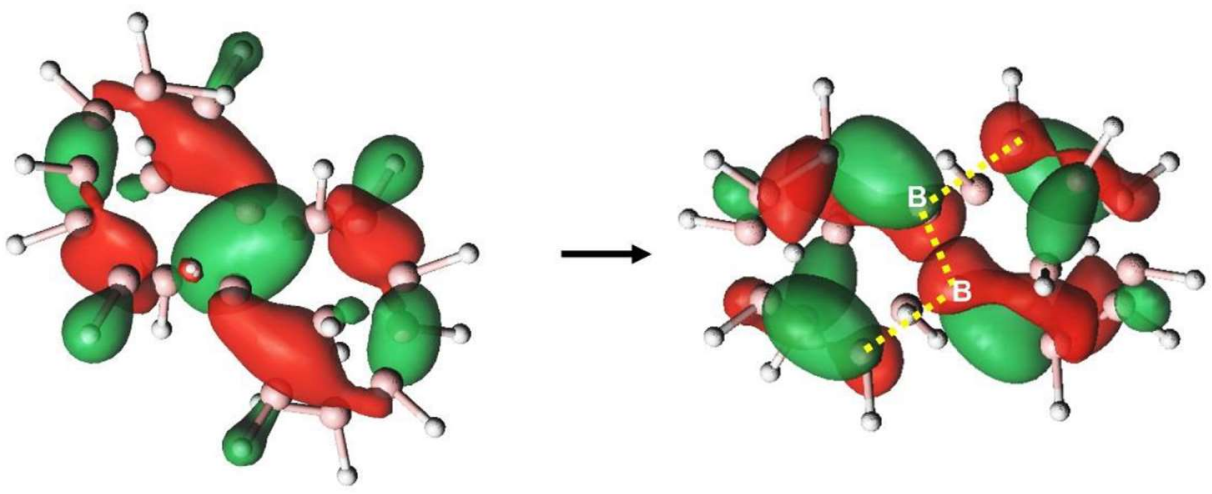

c)
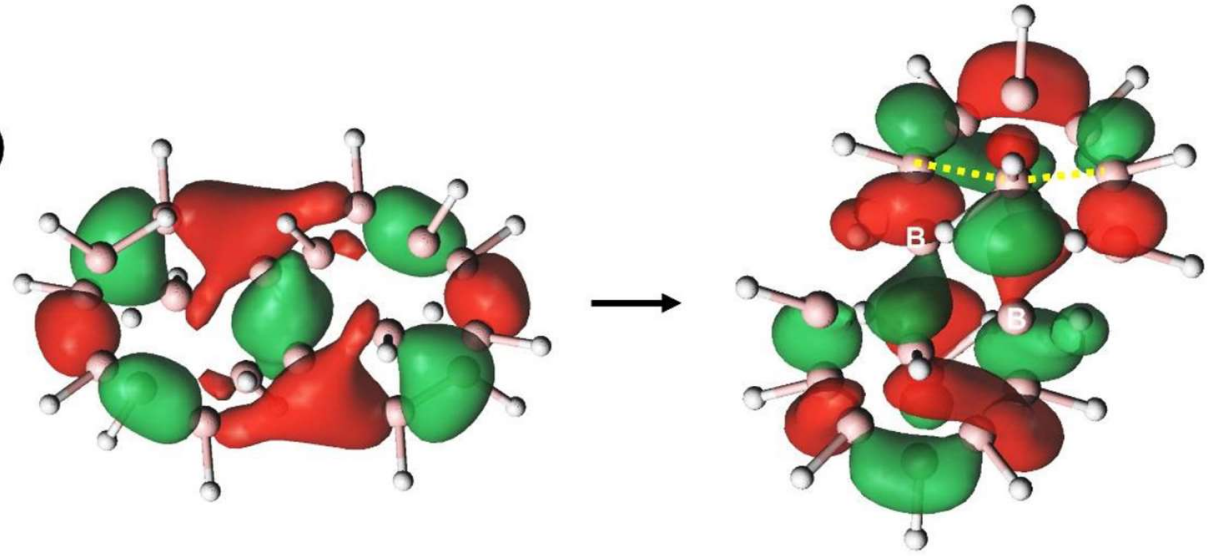

d)
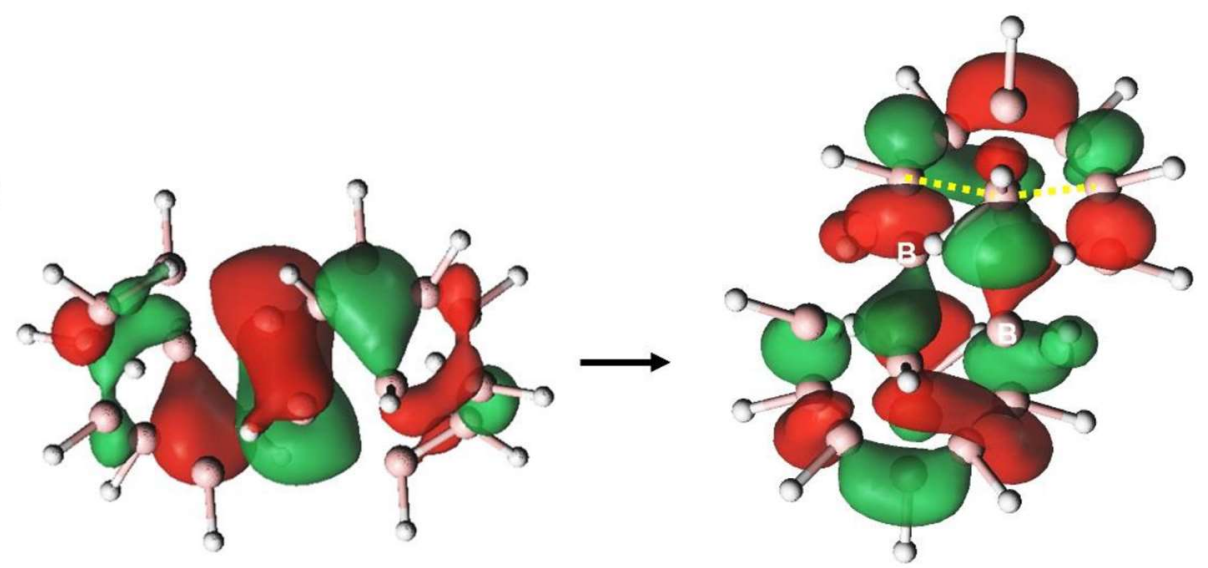
Figure S15. ${ }^{11} \mathrm{~B}-\left\{{ }^{1} \mathrm{H}\right\}$ spectrum of anti- $\mathrm{B}_{18} \mathrm{H}_{22}$ sample irradiated for a period of 270 minutes

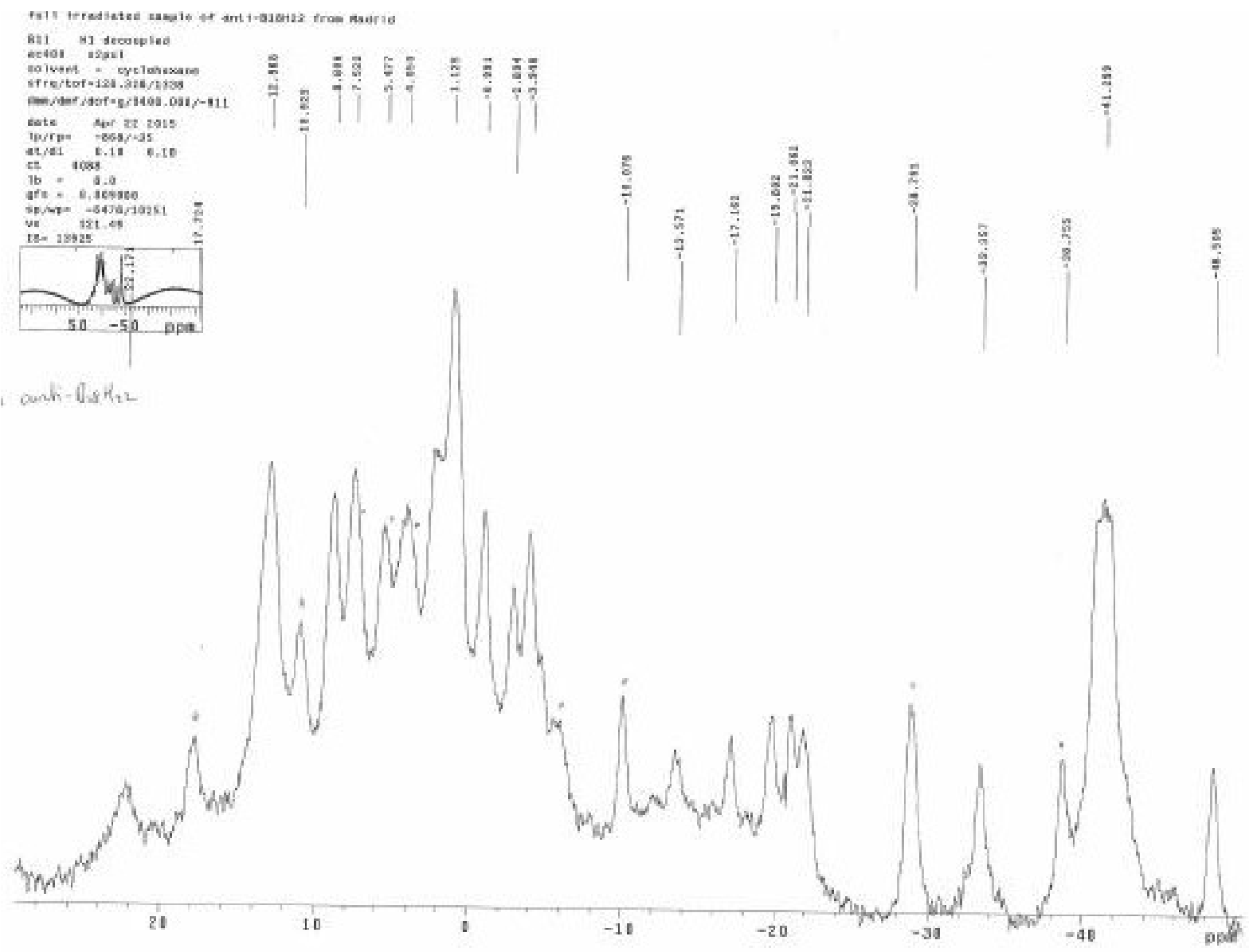


Figure S16. Mass spectrum of of anti- $\mathrm{B}_{18} \mathrm{H}_{22}$ sample irradiated for a period of 270 minutes

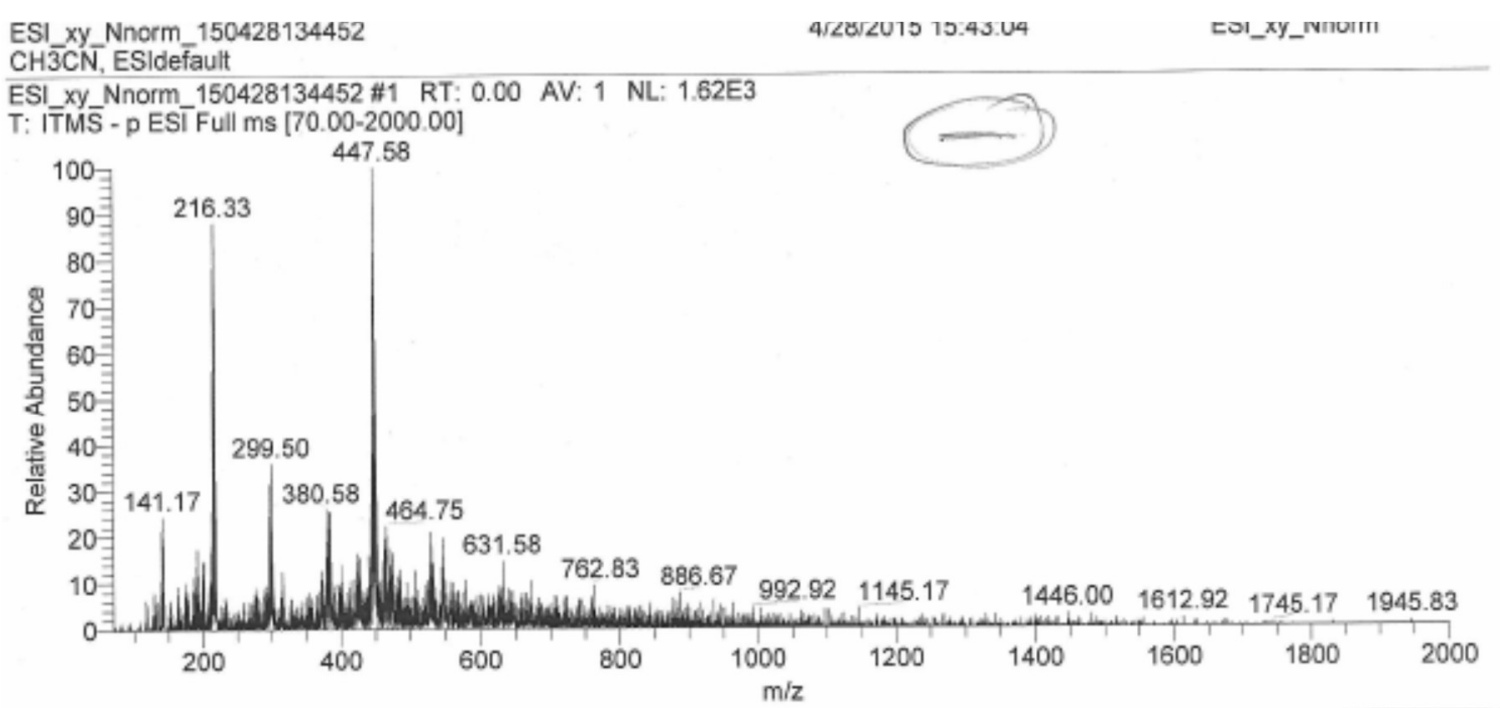

ESI_xy_Nnorm_150428134452 \#1 RT: 0.00 AV: 1 NL: $1.43 E 3$

T: ITMS - p EST' Full ms [70.00-2000.00]

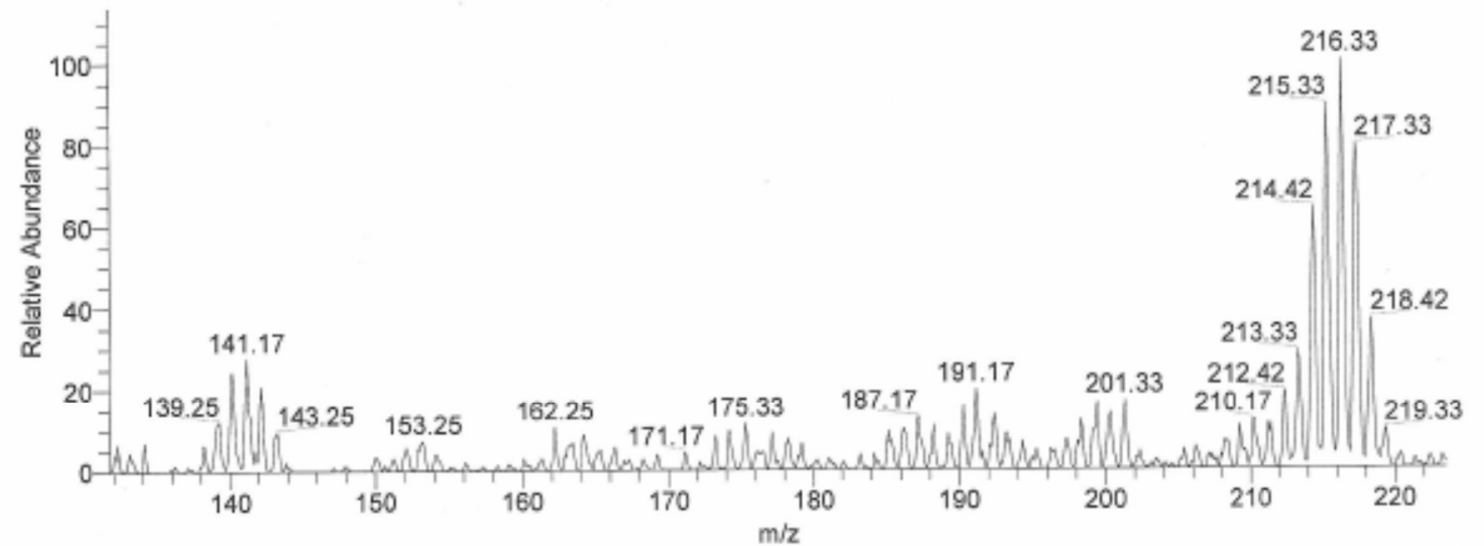

ESI_xy_Nnorm_150428134452 \#1 RT: 0.00 AV: 1 NL: $1.62 E 3$

T: ITMS - p ESĪ Full ms [70.00-2000.00]

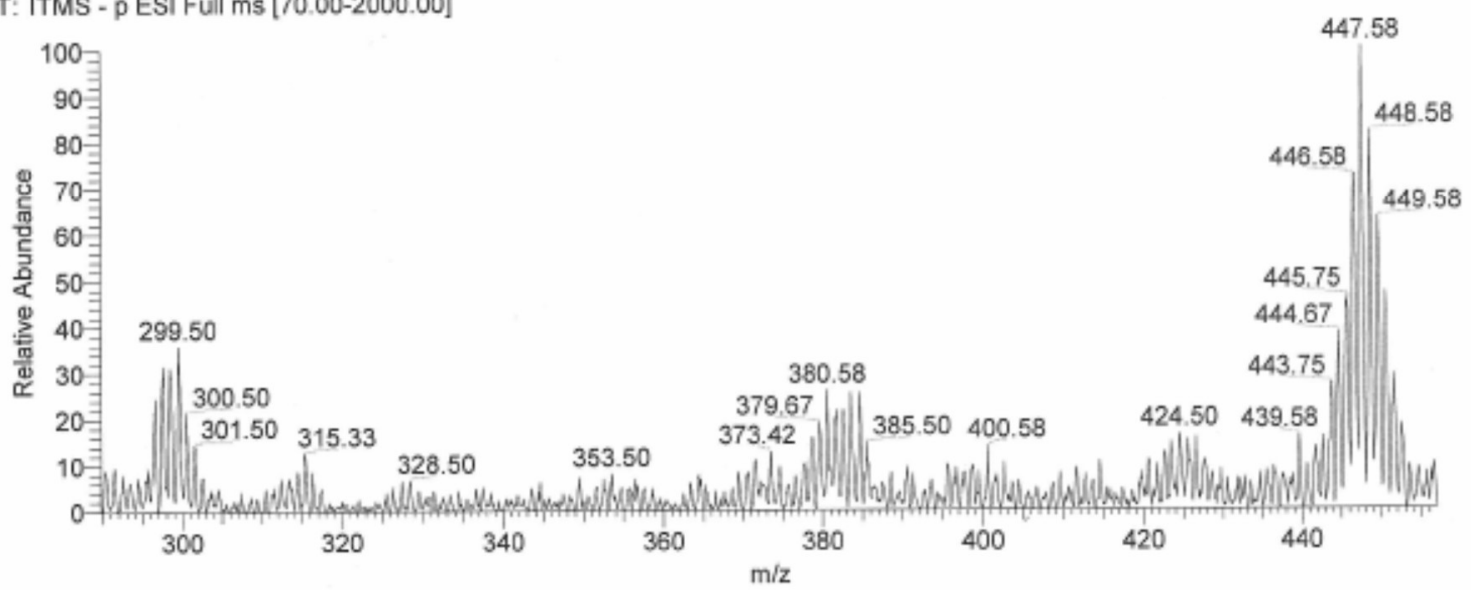


Figure S17. Reference (left-hand side) and 270 minute irradiated (right-hand side) samples of anti- $\mathrm{B}_{18} \mathrm{H}_{22}$ in $c$-hexane under UV lamp irradiation.

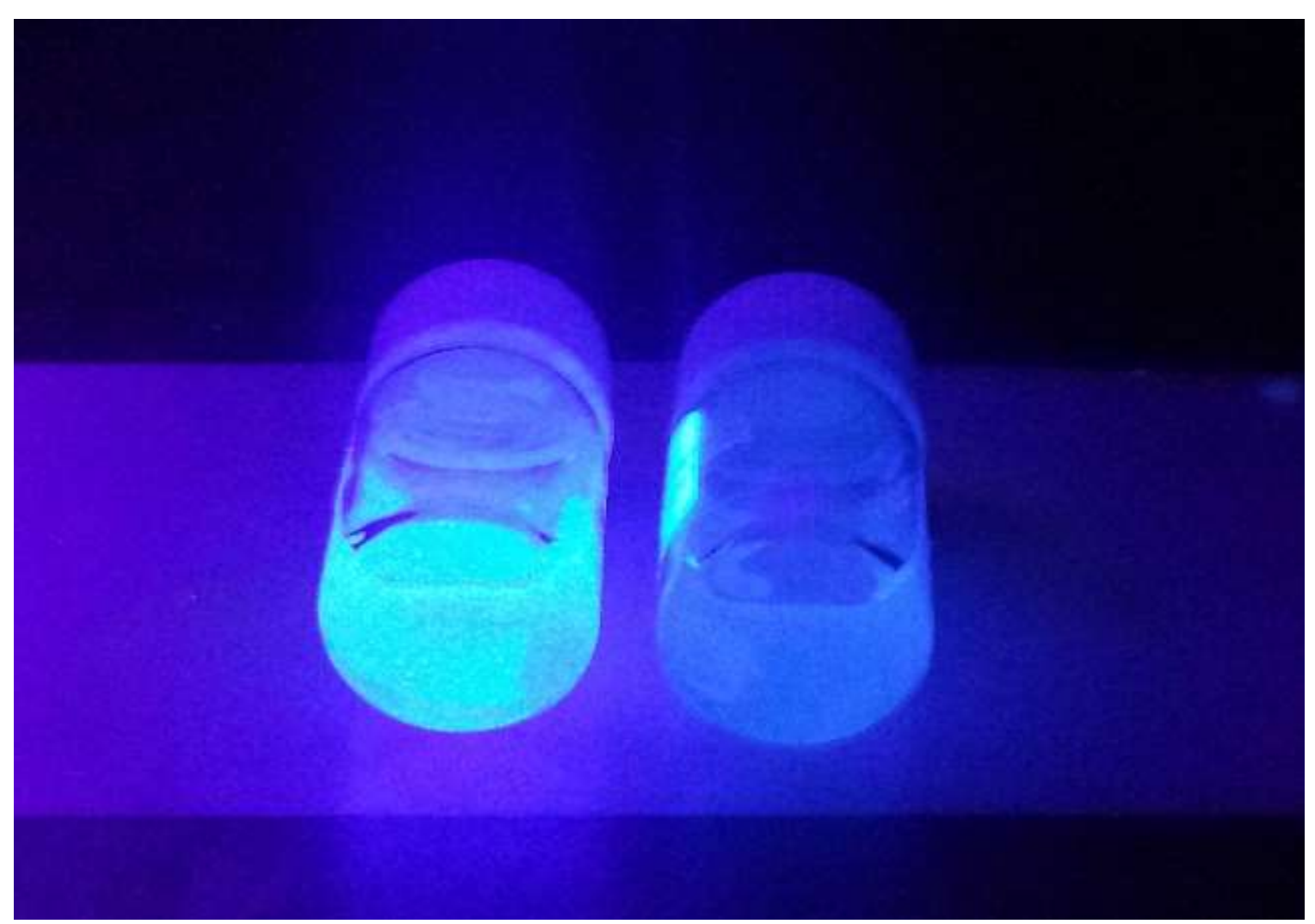




\section{Table of Contents (TOC)}

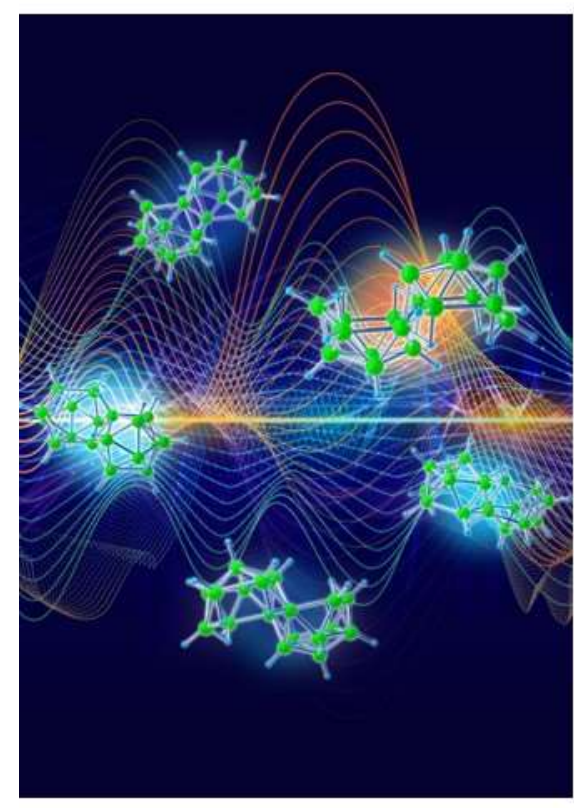

Unveiling the role of upper excited states in the photochemistry and laser performance of anti- $\mathbf{B}_{18} \mathbf{H}_{22}$.

L. Cerdán*, A. Francés-Monerris, D. Roca-Sanjuán*, J. Bould, J. Dolansky, M. Fuciman, and $M$. G. S. Londesborough*

The laser performance of anti- $\mathrm{B}_{18} \mathrm{H}_{22}(\sim 10 \%$ efficiency) does not match the expectations based on its exceptional photophysical properties $\left(\Phi_{F}=\right.$ 0.97 and high photostability). To understand this contradiction, we combine computational quantum chemistry, laser and UV-vis spectroscopy, NMR spectroscopy, as well as Mass spectrometry, to unveil the role of the upper excited states on the optical performance of this remarkable molecule. 